ESAIM: M2AN 49 (2015) 39-67

DOI: $10.1051 / \mathrm{m} 2 \mathrm{an} / 2014024$
ESAIM: Mathematical Modelling and Numerical Analysis

www.esaim-m2an.org

\title{
DEVELOPMENT AND STABILITY ANALYSIS OF THE INVERSE LAX-WENDROFF BOUNDARY TREATMENT FOR CENTRAL COMPACT SCHEMES *
}

\author{
FRANÇOIS VILAR ${ }^{1}$ AND CHI-WANG SHU ${ }^{1}$
}

\begin{abstract}
In this paper, we generalize the so-called inverse Lax-Wendroff boundary treatment [S. Tan and C.-W. Shu, J. Comput. Phys. 229 (2010) 8144-8166] for the inflow boundary of a linear hyperbolic problem discretized by the recently introduced central compact schemes [X. Liu, S. Zhang, H. Zhang and C.-W. Shu, J. Comput. Phys. 248 (2013) 235-256]. The outflow boundary is treated by the classical extrapolation and a stability analysis for the resulting scheme is provided. To ensure the stability of the considered schemes provided with the chosen boundaries, the G-K-S theory [B. Gustafsson, H.-O. Kreiss and A. Sundström, Math. Comput. 26 (1972) 649-686] is used, first in the semidiscrete case then in the fully discrete case with the third-order TVD Runge-Kutta time discretization. Afterwards, due to the high algebraic complexity of the G-K-S theory, the stability is analyzed by visualizing the eigenspectrum of the discretized operators. We show in this paper that the results obtained with these two different approaches are perfectly consistent. We also illustrate the high accuracy of the presented schemes on simple test problems.
\end{abstract}

Mathematics Subject Classification. 65M12.

Received November 10, 2013. Revised April 26, 2014.

Published online January 12, 2015.

\section{INTRODUCTION}

In numerical simulation of numerous physical phenomena, the choice in the scheme used is crucial. For example in aeroacoustics, the flows that generate noises are nonlinear, unsteady and usually turbulent. This aerodynamic noise is broadband with a fairly wide spectrum. Furthermore, the amplitudes of the physical variables of the aerodynamic noise are far smaller than those of the mean flow, and the distance from the noise source to the location of interest in aeroacoustic problems is quite long. Regarding these remarks, to ensure that the computed solution is uniformly accurate over such a long propagation distance, the numerical scheme should have minimal numerical dispersion and dissipation, or in other words should have a good wave resolution and high order accuracy. A relevant choice is the class of compact schemes $[3,12,15,18]$. Compared to the spectral methods, compact schemes can handle non-periodic boundary conditions a lot more easily, and usually have

Keywords and phrases. Central compact schemes, initial boundary value problem, inverse Lax-Wendroff, extrapolation, G-K-S theory, eigenvalue spectrum.

* Research supported by NASA grant NNX12AJ62A.

1 Division of Applied Mathematics, Brown University, Providence, RI 02912, USA.

francois_vilar@brown.edu; shu@dam.brown.edu 
much smaller numerical dispersion and dissipation errors than finite difference schemes of the same order of accuracy on the same mesh. In this paper, we study the particular case of the central compact schemes (CCS) introduced recently in [14], but the procedure and analysis can be easily generalized to other compact schemes and general finite difference schemes.

Even though the stencil of compact schemes is usually less wide than for finite difference methods of the same order of accuracy, particular treatments near the boundaries are still required. In the present work, the values of the points lying outside the computational domain, also named ghost points, are evaluated through different procedures: a classical Lagrangian extrapolation at the outflow boundary, and an inverse Lax-Wendroff (ILW) method at the inflow boundary. The latter one has been introduced in the case of conservation laws for weighted essentially non-oscillatory (WENO) finite difference schemes in [23], based on earlier works in $[5,6]$, and a simplified version has been given in [24]. We first extend the two boundary treatments to the case of compact schemes. Then, the stability issue is addressed.

The stability problem is a classical feature of numerical schemes, and thus has been tackled numerous times during the past decades. One of the most powerful tool for stability analysis on finite domain might be the normal mode analysis, which is based on the Laplace transform. The general stability theory based on this technique was given in [9]. This is the so-called G-K-S theory. In [9], the stability of fully discrete difference schemes are analyzed for initial boundary value problems (IBVP). Later on, the semidiscrete case has been tackled in [22]. In the present work, the normal mode analysis is used to ensure stability of central compact schemes provided with the considered boundary conditions. This study is done for different orders of accuracy. Due to the high complexity of the algebra involved using G-K-S theory in the case of very high order of accuracy, the stability is also addressed by visualizing the eigenvalue spectrum of the discretized operators. At the end, we briefly discuss about energy stability of the considered schemes and boundary treatments.

This paper is organized as follows. Section 2 presents the central compact schemes studied. Section 3 contains the definition of the different boundary procedures to define the ghost point values. In Section 4, G-K-S theory is used to address the stability of the central compact schemes provided with the presented boundary conditions in the simple case of initial boundary value linear hyperbolic equations. Eigenvalue spectrum of the discretized operators are analyzed in Section 5. We show that in the considered cases both methods, G-K-S and eigenspectrum analysis, exhibit perfectly consistent results, in the semidiscrete and fully discrete cases. Finally, using the energy method we show that the inverse Lax-Wendroff inflow boundary treatment maintains the stable and conservative behavior of the inner scheme, and mimics naturally continuous level results. We also illustrate the relevancy of the stability analysis done as well as the high accuracy of the presented schemes on simple test problems.

\section{Central compact schemes}

In this section, we present the methodology to design central compact schemes (CCS), see [14], in the simple case of conservation laws. Thus, we consider numerical approximations to the solution of the following problem

$$
\frac{\partial u}{\partial t}+\frac{\partial f(u)}{\partial x}=0
$$

A semidiscrete finite difference scheme can be represented as

$$
\left(\frac{\partial u}{\partial t}\right)_{i}=-f(u)_{i}^{x}
$$

where $f(u)_{i}^{x}$ is the approximation of $\frac{\partial f(u)}{\partial x}$ at the grid node $x_{i}$. 
The starting point to design these methods was the use of Lele's compact schemes. In [13], Lele proposed two kinds of compact schemes. One is a linear cell-centered compact scheme (CCCS) given by

$$
\begin{gathered}
\beta f_{i-2}^{x}+\alpha f_{i-1}^{x}+f_{i}^{x}+\alpha f_{i+1}^{x}+\beta f_{i+2}^{x} \\
a \frac{f_{i+\frac{1}{2}}-f_{i-\frac{1}{2}}}{\Delta x}+b \frac{f_{i+\frac{3}{2}}-\overline{f_{i-\frac{3}{2}}}}{3 \Delta x}+c \frac{f_{i+\frac{5}{2}}-f_{i-\frac{5}{2}}}{5 \Delta x} .
\end{gathered}
$$

The other is a cell-node compact scheme (CNCS) given by

$$
\begin{gathered}
\beta f_{i-2}^{x}+\alpha f_{i-1}^{x}+f_{i}^{x}+\alpha f_{i+1}^{x}+\beta f_{i+2}^{x} \\
a \frac{f_{i+1}-f_{i-1}}{2 \Delta x}+b \frac{f_{i+2}-f_{i-2}}{4 \Delta x}+c \frac{f_{i+3}-f_{i-3}}{6 \Delta x} .
\end{gathered}
$$

The left hand side of equations (2.3) and (2.4) contain the spatial derivatives $f_{i}^{x}$ at grid nodes, while the right hand side of equation (2.3) contains the cell-centered values $f_{i+\frac{1}{2}}$ at the center $x_{i+\frac{1}{2}}=\frac{1}{2}\left(x_{i}+x_{i+1}\right)$ of the cell $I_{i}=\left[x_{i}, x_{i+1}\right]$. The right hand side of equation (2.4), on the other hand, only contains the function values $f_{i}$ at the grid node $x_{i}$. The constraints on the coefficients $\alpha, \beta$ and $a, b, c$ corresponding to different orders of accuracy can be derived by matching the Taylor series coefficients and these have been listed in [13]. Lele showed that the resolution of cell-centered compact schemes is much better than the cell-node compact schemes. In this case, the stencil contains both grid points $x_{i}$ and half grid points $x_{i+\frac{1}{2}}$. However, only the values at the cell-centers are used to calculate the derivatives at the cell-nodes. The idea of CCS was so, if the values at both grid and half grid points are used, one could get a compact scheme with higher order of accuracy and better resolution. Thus, the central compact schemes write

$$
\begin{gathered}
\beta f_{i-2}^{x}+\alpha f_{i-1}^{x}+f_{i}^{x}+\alpha f_{i+1}^{x}+\beta f_{i+2}^{x} \\
a \frac{f_{i+\frac{1}{2}}-f_{i-\frac{1}{2}}}{\Delta x}+b \frac{f_{i+1}-f_{i-1}}{2 \Delta x}+c \frac{f_{i+\frac{3}{2}}-f_{i-\frac{3}{2}}}{3 \Delta x}+d \frac{f_{i+2}-f_{i-2}}{4 \Delta x}+e \frac{f_{i+\frac{5}{2}}-f_{i-\frac{5}{2}}}{5 \Delta x} .
\end{gathered}
$$

We note that the Lele's CCCS (2.3) and CNCS (2.4) are both special cases of this class of central compact schemes. These schemes contain the values of the cell-centers, which are unknown. A way to compute these unknowns is the use of an interpolation procedure, for example the high order compact interpolation proposed by Lele in [13]. However, doing so can introduce transfer errors, and thus reduce the resolution for high wave numbers. In [14] to overcome this drawback, the cell-center values are stored as independent computational variables and are evaluated using the same scheme as that for the cell nodes, by simply shifting the indices in (2.5) by $\frac{1}{2}$. Noticed that, this change brings increased memory requirement for storing cell center values, but it does not increase the computational cost, at least in one-dimension, since the compact interpolation is replaced by the compact scheme updating of comparable cost.

For the sake of simplicity, these central compact schemes can be rewritten as cell-node compact schemes on a twice more refined grid as

$$
\begin{gathered}
\beta f_{i-4}^{x}+\alpha f_{i-2}^{x}+f_{i}^{x}+\alpha f_{i+2}^{x}+\beta f_{i+4}^{x} \\
a \frac{f_{i+1}-f_{i-1}}{2 \Delta x}+b \frac{f_{i+2}-f_{i-2}}{4 \Delta x}+c \frac{f_{i+3}-f_{i-3}}{6 \Delta x}+d \frac{f_{i+4}-f_{i-4}}{8 \Delta x}+e \frac{f_{i+5}-f_{i-5}}{10 \Delta x} .
\end{gathered}
$$

The relationships among the coefficients $\alpha, \beta$ and $a, b, c, d, e$ in equation (2.5) or (2.6) are derived by matching the Taylor series coefficients of various orders. It leads to three families of central compact schemes: the explicit ones (CCS-E) for which $\alpha=0$ and $\beta=0$, the tridiagonal ones (CCS-T) where $\beta=0$, and the most generic pentadiagonal ones (CCS-P). In Figure 1, an example of the high accuracy and very low dissipation error of the 


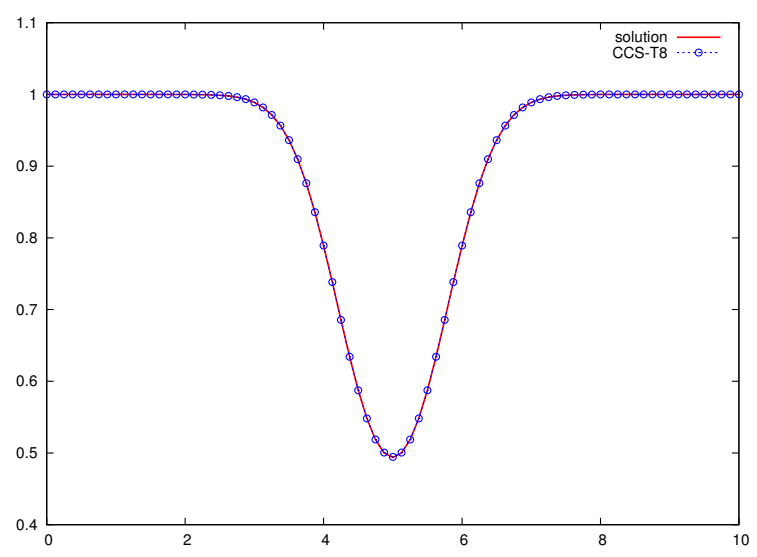

(a) Final time $t=50$

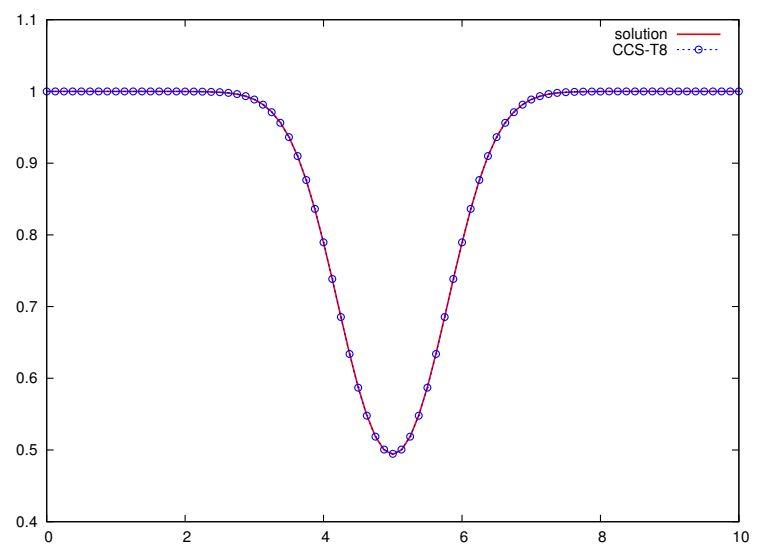

(b) Final time $t=200$

Figure 1. The distribution of the density along $x=5$ for the two dimensional advection of an isentropic vortex on a $80 \times 80$ Cartesian grid, with the RK3-CCST8 scheme.

central compact schemes is given. These numerical results have been obtained in the two-dimensional case of the Euler equations with the eighth-order scheme CCS-T8 provided with the third-order TVD Runge-Kutta time discretization, and correspond to the two dimensional advection of an isentropic vortex, with periodic boundary conditions.

Obviously, due to the relatively large stencil of central compact schemes, special treatments of points near the boundaries are required in the case of non-periodic boundary conditions. In [14], the issue of non-periodic boundary conditions is only discussed briefly with one set of particular boundary treatments which is found to be stable experimentally. In the next section, we present the boundary procedures to be studied in this paper, for the two cases of inflow and outflow boundaries respectively.

\section{BOUNDARY TREATMENTS}

To present the particular boundary treatments studied, we consider the simple scalar conservation initial boundary value problem (IBVP)

$$
\begin{cases}\frac{\partial u}{\partial t}+\frac{\partial f(u)}{\partial x}=0, & x \in\left[x_{A}, x_{B}\right], t \geq 0, \\ u\left(x_{A}, t\right)=g(t), & t \geq 0, \\ u(x, 0)=u_{0}(x), & x \in\left[x_{A}, x_{B}\right],\end{cases}
$$

where $g(t)$ is the prescribed boundary condition and $u_{0}(x)$ the initial solution. We assume that $f^{\prime}\left(u\left(x_{A}, t\right)\right)>0$ and $f^{\prime}\left(u\left(x_{B}, t\right)\right)>0$, where $f^{\prime}(u)=\frac{\mathrm{d} f(u)}{\mathrm{d} u}$, for $t \geq 0$. This assumption guarantees the left boundary $x=x_{A}$ is an inflow boundary where a boundary condition is needed, and the right boundary $x=x_{B}$ is an outflow boundary where no boundary condition is needed.

Now, let us discretize the domain $\left[x_{A}, x_{B}\right]$ by a uniform mesh $\left\{x_{j}\right\}_{j=0, \ldots, n}$ such as

$$
x_{0}-C_{A} \Delta x=x_{A} \leq x_{0}<x_{1}<\ldots<x_{n} \leq x_{B}=x_{n}+C_{B} \Delta x,
$$

where $C_{A} \in[0,1)$ and $C_{B} \in[0,1)$. 
Notice that the grid points $x_{0}$ and $x_{n}$ are not necessarily located on the boundaries $x_{A}$ and $x_{B}$, which is chosen on purpose since it is usually not possible to align boundary with grid points in a two-dimensional domain with complex geometries.

We note that the scheme definition (2.6) applied on points close to the boundaries involve the contribution of points outside the considered domain, called ghost points. Thus, we have to determine in a consistent manner and with an appropriate order of approximation these ghost points values.

\subsection{Outflow boundary}

Near the outflow boundary $x_{B}$, the ghost point values $f_{n+p}$, for $p=1, \ldots, 5$ and $f_{n+p}^{x}$, for $p=1, \ldots, 4$ have to be determined with suitable order of approximation $d$. It has been proven in [4] that outflow boundary extrapolation, which complements stable finite difference scheme, maintains stability for linear hyperbolic initial value problems. Thus, for the outflow boundary an extrapolation procedure is used. In order to evaluate the ghost values $f_{n+p}$, let us introduce the following definition

$$
f_{n+p}=f\left(u_{n+p}\right),
$$

where the $u_{n+p}$ are defined through the use of a Lagrangian extrapolation procedure of the appropriate order as follows

$$
u_{n+p}=P_{d}\left(x_{n+p}\right)=\sum_{j=1}^{d} u_{n-d+j} \prod_{\substack{l=1 \\ l \neq j}}^{d}\left(\frac{p+d-l}{j-l}\right)
$$

where $P_{d}(x)$ is the Lagrange polynomial of degree $(d-1)$ satisfying $P_{d}\left(x_{n-d+j}\right)=u_{n-d+j}$, for $j=1, \ldots, d$. An equivalent way to do the extrapolation is the use of a Taylor expansion

$$
u_{n+p}=\sum_{k=0}^{d-1} \frac{\left(p-C_{B}\right)^{k}}{k !} \Delta x^{k} u^{*(k)},
$$

where $u^{*(k)}=\left.\frac{\partial^{k} P_{d}}{\partial x^{k}}\right|_{x=x_{B}}$ is a $(d-k)^{\text {th }}$ order approximation of $\left.\frac{\partial^{k} u}{\partial x^{k}}\right|_{x=x_{B}}$.

We will see in the next section that this formulation is consistent with the one used in the inverse Lax-Wendroff procedure for the inflow boundary condition. Though, on contrary to formula (3.4), the extrapolation procedure (3.5) seems to depend on $C_{B}$. Actually, after some simple algebra one can find that these two formulations are perfectly equivalent and do not depend on the position of the boundary $x_{B}$.

Now, we extend this boundary extrapolation procedure to the case of compact schemes defining $f_{n+p}^{x}$ as

$$
f_{n+p}^{x}=\left.f^{\prime}\left(u_{n+p}\right) \frac{\partial u}{\partial x}\right|_{x=x_{n+p}},
$$

where $\left.\frac{\partial u}{\partial x}\right|_{x=x_{n+p}}$ will be evaluate through an extrapolation as follows

$$
\left.\frac{\partial u}{\partial x}\right|_{x_{n+p}}=\left.\frac{\partial P_{d}}{\partial x}\right|_{x_{n+p}}=\frac{1}{\Delta x} \sum_{j=1}^{d} u_{n-d+j} \prod_{\substack{l=1 \\ l \neq j}}^{d}\left(\frac{p+d-l}{j-l}\right) \sum_{\substack{q=1 \\ q \neq j}}^{d}\left(\frac{1}{p+d-q}\right) .
$$

Thanks to formulas (3.4) and (3.7), we are now able to express all the quantities present in the scheme definition (2.5), applied to points near the outflow boundary through the inner points. A similar procedure is also required at inflow boundary. 


\subsection{Inflow boundary}

As for the outflow boundary, the ghost points values $f_{-p}$, for $p=1, \ldots, 5$ and $f_{-p}^{x}$, for $p=1, \ldots, 4$ near the inflow boundary $x_{A}$, have to be determined with the appropriate order of approximation. Here, we present two different versions of the inverse Lax-Wendroff inflow boundary condition.

\subsubsection{Inverse Lax-Wendroff (ILW) procedure}

This procedure has been introduced in [23], and provided to finite difference schemes the ghost values for an inflow boundary using an arbitrary order of approximation and consistent with the boundary condition $g(t)$. In a similar manner to the outflow treatment, let us set

$$
f_{-p}=f\left(u_{-p}\right),
$$

where $u_{-p}$ are evaluated using a Taylor expansion as follows

$$
u_{-p}=\sum_{k=0}^{d-1} \frac{\left(-p+C_{A}\right)^{k}}{k !} \Delta x^{k} u^{*(k)},
$$

where $u^{*(k)}$, the $(d-k)^{\text {th }}$ order approximation of $\left.\frac{\partial^{k} u}{\partial x^{k}}\right|_{x=x_{A}}$, are this time evaluated by an ILW procedure, see [23]. Practically, we make use of the PDE, (3.1), and the boundary condition, $u\left(x_{A}, t\right)=g(t)$, to convert the spatial derivatives into time derivatives, and so determine the successive "moments" $u^{*(k)}$. For example, the first few moments would write

$$
\begin{aligned}
& u^{*(0)}=u\left(x_{A}, t\right)=g(t), \\
& u^{*(1)}=\left.\frac{\partial u}{\partial x}\right|_{x=x_{A}}=-\frac{g^{\prime}(t)}{f^{\prime}(g(t))}, \\
& u^{*(2)}=\left.\frac{\partial^{2} u}{\partial x^{2}}\right|_{x=x_{A}}=\frac{f^{\prime}(g(t)) g^{\prime \prime}(t)-2 f^{\prime \prime}(g(t)) g^{\prime}(t)^{2}}{f^{\prime}(g(t))^{3}} .
\end{aligned}
$$

Here, we extend this procedure to compact schemes, setting

$$
f_{-p}^{x}=\left.f^{\prime}\left(u_{-p}\right) \frac{\partial u}{\partial x}\right|_{x=x_{-p}} .
$$

In this last expression, we see the contribution of $u_{-p}$ which has already been determined by the ILW procedure. A Taylor expansion of the function $\frac{\partial u}{\partial x}$ at the boundary leads to

$$
\left.\frac{\partial u}{\partial x}\right|_{x=x_{-p}}=\sum_{k=0}^{d-2} \frac{\left(-p+C_{A}\right)^{k}}{k !} \Delta x^{k} u^{*(k+1)},
$$

where the $u^{*(k)}$ have already been computed in the evaluation of $u_{-p}$.

It has been proven in [23] that this inflow boundary treatment maintains stability of stable finite difference schemes in the case of linear hyperbolic initial value problems. We will show in the next section that this stability result holds in the case of central compact schemes as well. But a drawback of this procedure is its high algebraic complexity. The algebra involved in the ILW procedure relies on the PDEs and thus can be very heavy for very-high order of approximation, and even worse in the case of fully nonlinear multi-dimensional systems of equations or for problems with source terms. This reason has motivated the simplification presented in [24]. In this paper, the authors present a simplified version of the inverse Lax-Wendroff boundary procedure in which only the first two moments $u^{*(0)}$ and $u^{*(1)}$ in the Taylor expansion are evaluated using the ILW. The successive ones are determined using an extrapolation procedure. In [24], this procedure has been shown to be numerically stable when applied on a fifth order WENO scheme. Nonetheless this particular boundary treatment cannot maintain stability for arbitrary order of accuracy. This is the reason that we present a generalized simplified version wherein the first $k_{d}$ leading terms (in [24] $k_{d}=2$ ) are determined through the inverse Lax-Wendroff procedure, where naturally we would like to take the smallest $k_{d}$ which can guarantee stability. 


\subsubsection{Simplified inverse Lax-Wendroff (SILW) procedure}

Let us recall the definition $f_{-p}=f\left(u_{-p}\right)$ and the Taylor expansion of the solution at the boundary

$$
u_{-p}=\sum_{k=0}^{d-1} \frac{\left(-p+C_{A}\right)^{k}}{k !} \Delta x^{k} u^{*(k)} .
$$

This time, only the first $k_{d}$ moments in (3.12) will be computed by the ILW procedure. For $k=k_{d}, \ldots, d-1$, the successive moments are defined through a Lagrangian extrapolation as $u^{*(k)}=\left.\frac{\partial^{k} P_{d}}{\partial x^{k}}\right|_{x=x_{A}}$, and thus (3.12) rewrites

$$
u_{-p}=\sum_{k=0}^{k_{d}-1} \frac{\left(-p+C_{A}\right)^{k}}{k !} \Delta x^{k} u_{i l w}^{*(k)}+\sum_{k=k_{d}}^{d-1} \frac{\left(-p+C_{A}\right)^{k}}{k !} \Delta x^{k} u_{e x t}^{*(k)},
$$

where the successive moments $u_{i l w}^{*(k)}$ identify the ones computed through the inverse Lax-Wendroff procedure and $u_{\text {ext }}^{*(k)}$ the ones defined by

$$
u_{e x t}^{*(k)}=\sum_{j=1}^{d} \frac{u_{j-1}}{\Delta x^{k}} \prod_{\substack{l=1 \\ l \neq j}}^{d}\left(\frac{1-C_{A}-l}{j-l}\right) \sum_{\substack{q_{1}=1 \\ q_{1} \neq j}}^{d}\left(\frac{1}{1-C_{A}-q_{1}}\right) \ldots \sum_{\substack{q_{k}=1 \\ q_{k} \neq j \\ q_{k} \neq j \\ q_{1}, \ldots, q_{k-1}}}^{d}\left(\frac{1}{1-C_{A}-q_{k}}\right) .
$$

For $f_{-p}^{x}=\left.f^{\prime}\left(u_{-p}\right) \frac{\partial u}{\partial x}\right|_{x=x_{-p}}$, a similar procedure is used which writes

$$
\left.\frac{\partial u}{\partial x}\right|_{x=x_{-p}}=\sum_{k=0}^{k_{d}-2} \frac{\left(-p+C_{A}\right)^{k}}{k !} \Delta x^{k} u_{i l w}^{*(k+1)}+\sum_{k=k_{d}-1}^{d-2} \frac{\left(-p+C_{A}\right)^{k}}{k !} \Delta x^{k} u_{e x t}^{*(k+1)} .
$$

In this section, all the ghost points contribution has been defined through the inner point variables. Thanks to the three different procedures discussed above corresponding to the inner scheme, the outflow and the inflow boundaries, respectively, we finally get the considered semidiscretization.

It has been proven that outflow extrapolation [4] and inflow inverse Lax-Wendroff [23] maintain stability of a Cauchy stable finite difference scheme. We extend this result to the central compact scheme case, and also analyze the stability of these schemes provided with the simplified inverse Lax-Wendroff inflow boundary treatment.

\section{StabiLity}

In this section we present the stability analysis of central compact schemes provided with extrapolation outflow boundary and inverse lax-Wendroff or its simplified version for the inflow boundary. We start this stability study using the G-K-S theory [9,22], firstly in the semidiscrete case then in the fully discrete case using the third-order TVD Runge-Kutta time discretization. Afterwards, due to the high complexity of the algebra involved in the G-K-S theory, the stability issue will be addressed via visualizing the eigenspectrum of the discretized operators. In the end, the results obtained with these two different approaches will be shown to be perfectly consistent.

\subsection{G-K-S theory}

A powerful technique was developed to assess the stability of numerical boundary conditions by Gustafsson, Kreiss and Sundström, based on the idea of normal mode analysis. This technique, generally referred to as the G-K-S theory, establishes conditions that the inner scheme and boundary schemes must satisfy to ensure 
stability. The specific details of the theory given in the fully discrete case in [9] and in the semidiscrete case in [22] can be found in numerous work and will not be detailed here. The essential elements of the propositions that form the basis of this work follow.

Proposition 4.1. G-K-S theory asserts that to show stability for the finite-domain problem, it is sufficient to show that the inner scheme is Cauchy stable on $(-\infty,+\infty)$, and that each of the two quarter-plane problems is stable with the use of normal mode analysis. Thus, the stability of the finite-domain problem is broken into the summation of three simpler problems.

Proposition 4.2. For each quarter-plane problem that arises from Proposition 4.1, a necessary and sufficient condition for stability of the IBVP is that no eigensolution exists. This proposition is true for either the fully discrete case [9], or the semidiscrete case [22].

The interested reader may find the original theorems and statements in the two seminal papers [9, 22], in which Proposition 4.1 refers to Theorem 5.4 in [9] and to the Section 3 statements in [22], while Proposition 4.2 refers to the Section 10 lemmas and theorems in [9] and to the "Main theorem" in [22].

Both Propositions 4.1 and 4.2 rely on a definition of an eigensolution for their quarter-plane analysis. In the remainder, the definition of an eigensolution is given in the case of the linear initial boundary value quarter-plane problem

$$
\begin{cases}\frac{\partial u}{\partial t}+A \frac{\partial u}{\partial x}=0, & x \geq 0, t \geq 0, \\ u(0, t)=g(t), & t \geq 0, \quad \text { if } A>0, \\ u(x, 0)=u_{0}(x), & x \geq 0, \\ \|u(., t)\|<\infty, & t \geq 0,\end{cases}
$$

where $\|u(., t)\|=\int_{0}^{\infty}|u(x, t)|^{2} \mathrm{~d} x$. We will use the same definition of the quarter-plane problem regardless the type of boundary considered, inflow or outflow. The difference will arise in the presence or not of a prescribed boundary condition $u(0, t)=g(t)$ depending on the sign of $A$, see (4.1). In (4.1), the right boundary has been removed and the boundary condition is substituted by the requirement that the $L_{2}$-norm is finite. We introduce now an uniform discretization of the domain, such as $0 \leq x_{0}=C_{0} \Delta x<x_{1}<\ldots$, where $\left\{x_{j}\right\}_{j=0,1, \ldots}$ are the grid points and $C_{0} \in[0,1)$ being related to the distance between the boundary $x=0$ and the first grid point $x_{0}$. We now consider a general definition of compact schemes inside the domain, far enough from the boundary

$$
\mathcal{P} \frac{\mathrm{d} u_{j}}{\mathrm{~d} t}=-\frac{A}{\Delta x} \mathcal{Q} u_{j}, \quad \text { for } j=r, r+1, \ldots
$$

where the different operators write

$$
\mathcal{P}=\sum_{i=-r_{L}}^{p_{L}} \alpha_{i} E^{i}, \quad \mathcal{Q}=\sum_{i=-r_{R}}^{p_{R}} a_{i} E^{i}, \quad E^{i} u_{j}=u_{j+i},
$$

and $r=\max \left(r_{L}, r_{R}\right)$. Obviously, near the boundary particular schemes are required. A generic definition of the boundary schemes is given in

$$
\mathcal{D}_{j} \frac{\mathrm{d} u_{j}}{\mathrm{~d} t}=-\frac{A}{\Delta x} \mathcal{B}_{j} u_{j}+\widetilde{g}_{j}(t), \quad \text { for } j=0,1, \ldots, r-1
$$

where $\mathcal{D}_{j}$ and $\mathcal{B}_{j}$ write

$$
\mathcal{D}_{j}=\sum_{i=-r_{L}^{j} \geq-j}^{p_{L}^{j}} d_{i}^{j} E^{i}, \quad \mathcal{B}_{j}=\sum_{i=-r_{R}^{j} \geq-j}^{p_{R}^{j}} b_{i}^{j} E^{i} .
$$

In (4.3), $\widetilde{g}_{j}(t)$ corresponds to the boundary contribution in the inflow boundary case. In the remainder, we first consider the semidiscrete case. 


\subsubsection{Semidiscrete case}

Hereon, for the semidiscretization defined previously, we give the following definition of an eigensolution:

Definition 4.3. An eigensolution for the IBVP defined by equations (4.2)-(4.3) is the nontrivial function $v(x, s)=\mathrm{e}^{s t} \phi(x)$, which satisfies

1) $s \Delta x \mathcal{P} v_{j}+A \mathcal{Q} v_{j}=0, \quad j=r, r+1, \ldots$

2) $s \Delta x \mathcal{D}_{j} v_{j}+A \mathcal{B}_{j} v_{j}=\widetilde{g}_{j}(t), \quad j=0,1, \ldots, r-1$.

3) $\operatorname{Re}(s) \geq 0$.

4) For $\operatorname{Re}(s)>0, v(x, s)$ is bounded as $x \rightarrow \infty$.

5) For $\operatorname{Re}(s)=0, v(x, s)=\lim _{\varepsilon \rightarrow 0^{+}} v(x, s+\varepsilon)$, where $v(x, s+\varepsilon)$ satisfies the conditions a) and c) with respect with $s+\varepsilon$ (generalized eigensolution).

Then, if one cannot find such a solution it proves the stability of the quarter-plane problem. Now to illustrate practically this stability analysis, we study the case of the sixth order tridiagonal central compact scheme CCST6. In this case, the numerical approximation of the spatial derivative, far enough from the boundary $(j \geq 2)$, is given by

$$
-\frac{u_{j-2}^{x}}{12}+u_{j}^{x}-\frac{u_{j+2}^{x}}{12}=\frac{16}{9} \frac{u_{j+1}-u_{j-1}}{2 \Delta x}-\frac{17}{18} \frac{u_{j+2}-u_{j-2}}{4 \Delta x} .
$$

Now, to ensure the existence or non-existence of eigensolutions normal mode analysis is performed, and thus a solution of the form $u_{j}(t)=\mathrm{e}^{s t} \phi_{j}$, where $\phi_{j}=\sigma K^{j}$ is assumed. Substituting this particular solution in the inner semidiscrete scheme produces the characteristic equation

$$
\widetilde{s}\left(K^{2}-\frac{1}{12}\left(K^{4}+1\right)\right)+\operatorname{sgn}(A)\left(\frac{16}{18}\left(K^{3}-K\right)-\frac{17}{72}\left(K^{4}-1\right)\right)=0,
$$

where $\operatorname{sgn}(A)$ gives the sign of $A$, and $\widetilde{s}=s \frac{\Delta x}{|A|}$ is the eigenvalue. The characteristic equation (4.5) has only two roots $K$ which yield $|K|<1$, for $\operatorname{Re}(\widetilde{s})>0$. The two other roots will become exponentially unbounded as $j$ becomes large and thus can be ignored. In the particular case where $\operatorname{Re}(\widetilde{s})=0$, the four roots of the characteristic equations are such that one yields $|K|>1$, another $|K|<1$ and the two last roots lie on the unit circle $|K|=1$. Thus, in this case three roots seem to be admissible in such way that $|K| \leq 1$. But actually, similarly to the previous case for which $\operatorname{Re}(\widetilde{s})>0$, only two roots have to be considered. Indeed, one of the root lying on the unit circle becomes exponentially unbounded as $\widetilde{s}$ gets perturbed in the right half-plane, and thus does not respect the condition 5) of Definition 4.3. For instance, in the case where $\widetilde{s}=0$ the three roots of equation (4.5) such that $|K| \leq 1$ are $K_{1}=1, K_{2}=-1$ and $K_{3}=\frac{32-7 \sqrt{15}}{17} \simeq 0.2876$. We have to determine if the first two are stable to perturbations or not. To do so we substitute $\widetilde{s}=\delta$ into the characteristic equation (4.5) which produces to first order $K_{1}=1+\delta$ and $K_{2}=-1+\frac{15}{49} \delta$, where only the latter lies inside the unit circle. This is the reason we can state that for any $\operatorname{Re}(\widetilde{s}) \geq 0$ the general solution to be considered has the form

$$
\phi_{j}=\sigma_{1} K_{1}^{j}+\sigma_{2} K_{2}^{j},
$$

where $K_{1}, K_{2}$ are the two roots with $|K| \leq 1$ and $\sigma_{1}, \sigma_{2}$ the two constants which remain to be determined.

It is clear in equation (4.4) that for the two first grid points $x_{0}$ and $x_{1}$, the value of points outside the domain has to be defined. The boundary procedures presented in the previous sections are used to do so. For example, in the outflow quarter-plan problem $(A=-1)$, extrapolation is used to define $u_{-p}$ and $u_{-p}^{x}$, for $p=1,2$. In the end, the particular schemes respectively on $j=0$ and $j=1$ write

$$
\begin{aligned}
& \widetilde{s}\left(72 \phi_{0}-6 \phi_{2}\right)+\frac{1647}{10} \phi_{0}-363 \phi_{1}+358 \phi_{2}-234 \phi_{3}+\frac{177}{2} \phi_{4}-\frac{71}{5} \phi_{5}=0, \\
& \widetilde{s}\left(72 \phi_{1}-6 \phi_{3}\right)+\frac{71}{5} \phi_{0}+\frac{159}{2} \phi_{1}-150 \phi_{2}+74 \phi_{3}-21 \phi_{4}+\frac{33}{10} \phi_{5}=0 .
\end{aligned}
$$


Substituting the general solution (4.6) in these boundary schemes yield a two-unknowns equation system, where the unknowns are the two constants $\sigma_{1}$ and $\sigma_{2}$. This system has only the trivial solution unless its determinant is null. Practically, we seek functions of the general form (4.6), with $\left|K_{1}\right| \leq 1$ and $\left|K_{2}\right| \leq 1$, solutions of the characteristic equation (4.5) in the case of a null determinant of the system deriving from the boundary schemes, (4.7) and (4.8). If no such solution exists, it proves the stability of the semidiscrete scheme on the considered quarter-plan problem. If one can find such solutions but only in the case where $\operatorname{Re}(\widetilde{s})<0$, according to Propositions 4.1 and 4.2 no eigensolution exists and thus it also proves stability. In the end, there are only two cases which exhibit instabilities. The first one is the case where such a solution exists with $\operatorname{Re}(\widetilde{s})>0$. The second one is the case where a solution exists with at least one of the roots on the unit circle and $\operatorname{Re}(\widetilde{s})=0$. In this specific situation, the scheme is unstable only if this particular solution is a generalized eigensolution, see Proposition 4.1. This last situation will be detailed immediately.

In the considered case of the outflow quarter-plan problem semidiscretization with the CCS-T6 scheme provided with extrapolation boundary, the only solutions of form (4.6) ensuring a null determinant of the boundary system belong to the case where $\widetilde{s}=0$ and one of the roots writes $K=1$. As shown previously this particular root becomes exponentially unbounded as $\widetilde{s}$ gets perturbed in the right half-plane. Thus these particular solutions are not eigensolutions of the IBVP under consideration. In the end, one can conclude that the central compact scheme CCS-T6 provided with extrapolation boundary is G-K-S stable. This last result holds for any central compact schemes. Consequently, using normal mode analysis and the G-K-S theory, we have proved that outflow extrapolation boundary maintains stability in the semidiscrete case of the central compact schemes, for any order of accuracy and for any position of the boundary $\left(C_{0} \in[0,1)\right)$.

The same analysis can be applied to the inflow quarter-plane problem $(A=1)$. This time, a boundary condition of the form $u(0, t)=g(t)$ has to be prescribed. For the purpose of the stability analysis, $g(t)$ can be set to zero without loss of generality. Under this assumption and using the inverse Lax-Wendroff boundary treatment, all the ghost point values are set to zero. In this case, the two boundary schemes respectively on $j=0$ and $j=1$ write

$$
\begin{aligned}
& \widetilde{s}\left(72 \phi_{0}-6 \phi_{2}\right)+64 \phi_{1}-17 \phi_{2}=0, \\
& \widetilde{s}\left(72 \phi_{1}-6 \phi_{3}\right)+64\left(\phi_{2}-\phi_{0}\right)-17 \phi_{3}=0 .
\end{aligned}
$$

In this situation, the normal mode analysis tells us that there is no non-trivial function which is a solution of both the characteristic equation (4.5) and the boundary schemes equations, (4.9) and (4.10), at the same time. Finally, one can prove that the inverse Lax-Wendroff treatment of the boundary also maintain stability in the semidiscrete case of the central compact schemes, for any order of accuracy and for any position of the boundary.

For the simplified inverse Lax-Wendroff procedure, the same analysis leads to the expected result that the stability of the semidiscretization depends on the number of leading term $k_{d}$ used, and on the position of the boundary. The aim is to determine the minimum number $k_{d}$ of leading terms in the SILW boundary procedure in order to ensure stability of central compact schemes. The same example as before (CCS-T6 scheme) is used to exhibit the approach used and the results obtained. In this case, we derive as previously the two boundary scheme equations, for $j=0$ and $j=1$. The system obtained will not be given explicitly here due to its high complexity. Obviously, these two equations depend directly on the boundary position $C_{0}$. The procedure was so to compute through the use of the software Mathematica the solutions of both characteristic equation (4.5) and of a null boundary system determinant, for any boundary position. In the end, we display the maximum real part of the corresponding eigenvalues $\widetilde{s}$, if such function exists, depending on $C_{0} \in(0,1]$, see Figure 2 .

In Figure 2, the case of one leading term is considered. One can see that for positions of the boundary where $C_{0} \in(0,0.503]$, the quarter-plane problem possesses solutions. Furthermore, the maximum of the real part of the corresponding eigenvalues is bigger than zero. This particular solution corresponds to an eigensolution, see Proposition 4.1, and according to Proposition 4.2 the semidiscrete scheme is not stable. For value of $C_{0}$ close to 0.5 , the problem does not exhibit solution. The scheme is then stable in this particular case. Finally, if the 


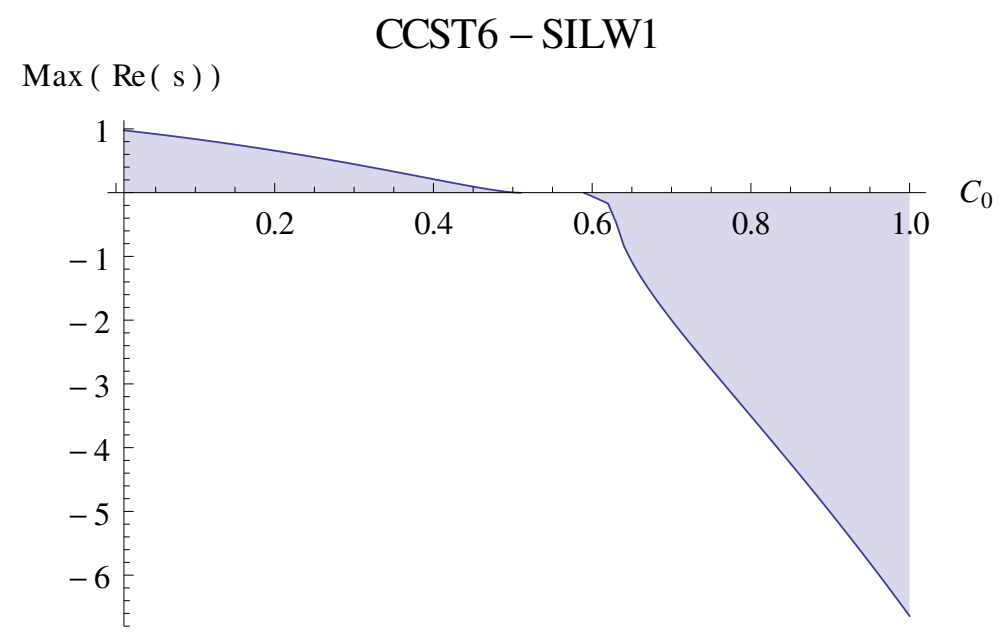

Figure 2. Maximum of the real part of the eigenvalues as function of $C_{0}$ for the CCS-T6 scheme with the SILW boundary condition with one leading term.

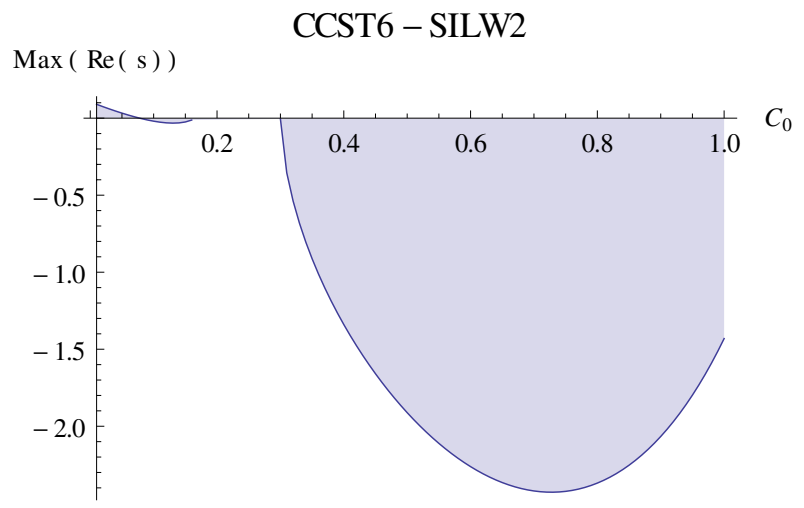

(a) Two leading term.

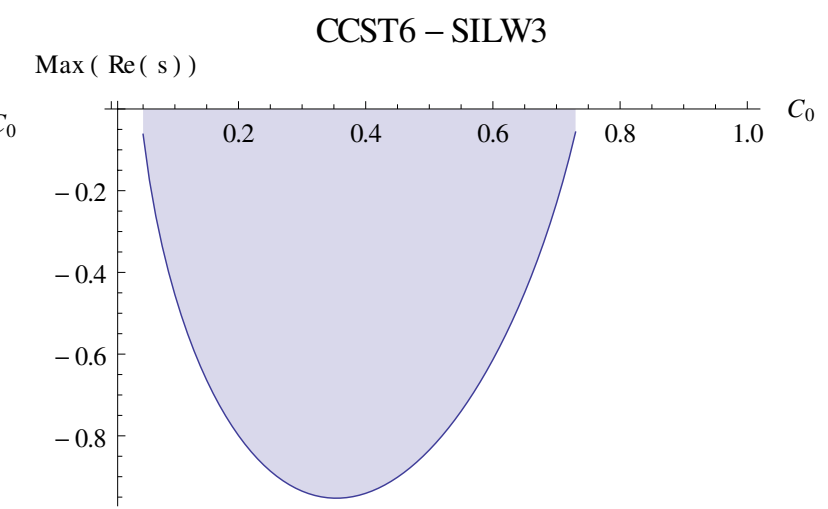

(b) Three leading terms.

Figure 3. Maximum of the real part of the eigenvalues as function of $C_{0}$ for the CCS-T6 scheme with the SILW boundary condition with two and three leading terms.

boundary is far enough from the first grid point, one can see on Figure 2 that the problem presents solutions, but for which the eigenvalues are less than zero in their real part. These solutions are not eigensolutions, and thus the scheme is stable for these boundary positions. In the end, the results displayed in Figure 2 show that one leading term in the simplified inverse Lax-Wendroff procedure is not enough to ensure stability of the CCS-T6 scheme for arbitrary position of the boundary. Consequently, we perform the same analysis in the two cases of two and three leading terms, see Figures $3 \mathrm{a}$ and $3 \mathrm{~b}$.

In Figure 3a, we can observe that with two leading terms, the semidiscrete scheme is still unstable in cases where the boundary and the first grid point are very close to each other. However, with three leading terms, see $3 \mathrm{~b}$, CCS-T6 scheme is perfectly stable for any position of the boundary. One can see that for some specific values of $C_{0} \in[0.05,0.73]$ the problem has nontrivial solutions but for which the real part of the eigenvalues is strictly less than zero. Performing the same analysis with more leading terms, one would find that for any values 
of $C_{0}$, the considered problem does not have nontrivial solutions, similar to the full ILW boundary condition. In the end, the CCS-T6 scheme requires at least three leading terms in the simplified inverse Lax-Wendroff boundary treatment to preserve stability for arbitrary position of the boundary.

The algebraic complexity involved in showing stability of the IBVP is dramatically reduced in the semidiscrete case, because time remains continuous. However, numerical stability is a fully discrete concept, and a connection between the semidiscrete and fully discrete stabilities must be used. The procedure presented here allows us to address the stability of semidiscrete schemes provided with specific boundary treatments. To ensure the relevance of the stability behaviors observed in the semidiscrete case, we now present the fully discrete extension of the previous analysis.

\subsubsection{Fully discrete case}

In the whole study presented, the choice has been made to use the well-known third-order TVD Runge-Kutta scheme, see [20], as time discretization (other time discretizations can certainly also be analyzed using the same techniques). We will first recall the stability domain of such method. To that purpose, let us consider the following general system

$$
\frac{\mathrm{d} u}{\mathrm{~d} t}=F(t, u) .
$$

To derive the eigenvalue problem, we set $F(t, u)=s u$. This relationship substituted in the RK3 discretization leads to the time discrete equation

$$
u^{n+1}=\left(1+\mu+\frac{\mu^{2}}{2}+\frac{\mu^{3}}{6}\right) u^{n}
$$

where $u^{n}=u\left(x, t^{n}\right)$ and $\mu=s \Delta t$, with $\Delta t$ the time step. Assuming a solution of the form $u^{n}=z^{n} u^{0}$, where $z$ is a complex number, the stability domain of the considered time discretization writes

$$
|z(\mu)| \leq 1, \quad \text { where } \quad z(\mu)=1+\mu+\frac{\mu^{2}}{2}+\frac{\mu^{3}}{6},
$$

where $z(\mu)$ is nothing but a third-order Taylor expansion of $\mathrm{e}^{\mu}$.

We recall that in the semidiscrete case, the normal mode analysis assumes a solution of form $u_{j}\left(t^{n+1}\right)=$ $\mathrm{e}^{s \Delta t} u_{j}\left(t^{n}\right)=\mathrm{e}^{\widetilde{s} \frac{\lfloor A \Delta t}{\Delta x}} u_{j}\left(t^{n}\right)$. The semidiscrete scheme is stable only if one cannot find an eigenvalue $\widetilde{s}$ lying in the right half-plane, i.e. $\operatorname{Re}(\widetilde{s})>0$, nor any generalized eigenvalue. In the fully discrete case, a solution of form $u_{j}^{n+1}=z(s \Delta t) u_{j}^{n}=z\left(\widetilde{s} \frac{|A| \Delta t}{\Delta x}\right) u_{j}^{n}$ has been assumed. The corresponding sufficient condition of stability would be the fully discrete scheme is stable only if one cannot find an eigenvalue $z$ lying outside the unit circle, i.e. $|z|>1$, nor any generalized eigenvalue. Obviously, if $z(\mu)$ is the exponential function, the semidiscrete and fully discrete stabilities are perfectly equivalent. But it is clear that the fully discrete case is just an approximation of the semidiscrete time continuous case, and for explicit Runge-Kutta time advancements $z(\mu)$ is nothing but a Taylor expansion of the exponential. Actually, the fully discrete scheme will be stable if the stability domain of the semidiscrete scheme lies in the one of the time discretization. This is the reason that it is possible for a scheme to be stable in its semidiscrete form but not stable after time discretization.

Practically, to demonstrate the stability of the considered schemes we substitute in the time discretization resolvent equation of $z$, equation (4.13), the eigenvalues $\widetilde{s}$ found previously in the semidiscrete G-K-S analysis. Then, one has to verify that there is no eigensolution of the quarter-plane problem. We note that $z$ depends on $\widetilde{s}$ and $\frac{|A| \Delta t}{\Delta x}$. We introduce here the CFL condition

$$
\lambda_{c f l}=\frac{|A| \Delta t}{\Delta x},
$$

where $\lambda_{c f l}>0$ is a scalar. 


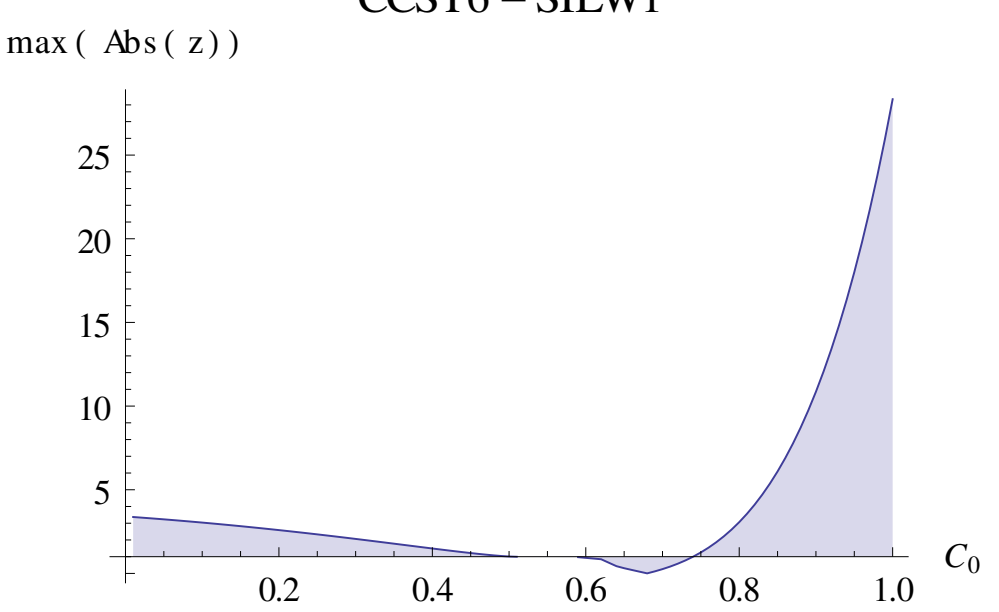

Figure 4. Maximum of the absolute value of the eigenvalues as function of $C_{0}$ for the RK3CCST6 scheme with the SILW boundary condition with one leading term and $\lambda_{c f l}=0.961$.

The fully discrete stability analysis is done at first with the same CFL condition as that for the periodic boundary case, i.e. condition ensuring that the inner scheme is Cauchy stable (in the RK3-CCST6 case, $\lambda_{c f l}=$ 0.961 is the maximum CFL condition ensuring the stability of the inner scheme). If the fully discrete scheme is not stable under this condition $(\exists z,|z|>1$ or a generalized eigenvalue), we use a decreasing sequence of CFL condition, re-performing at each step the stability analysis. The decreasing sequence stops as soon as the scheme has proved to be stable, or when $\lambda_{c f l}$ goes to zero.

Finally, using such a procedure we have proved that outflow extrapolation boundary as well as inverse Lax-Wendroff treatment of the inflow boundary maintain stability in the fully discrete case of central compact schemes provided with third-order TVD Runge-Kutta, for any order of accuracy and any position of the boundary, and under the same CFL condition as that for the periodic boundary case. For the simplified inverse Lax-Wendroff procedure, the stability depends as before on the number of leading terms used. We give here the same example as in the semidiscrete analysis. In the different figures displayed, see Figures 4 and 5 , the $C_{0}$-axis is now located at $|z|=1$. In this configuration, consistent with the semidiscrete case, existence of eigenvalue above the $C_{0}$-axis proves instability.

In Figure 4, the case of one leading term is considered. One can see that, consistent with the semidiscrete case, if the boundary is too close to the first grid point the fully discrete scheme is not stable. For value of $C_{0}$ close to 0.5 , the quarter-plane problem does not exhibit solution. Nevertheless, if $C_{0} \in[0.742,1]$ the fully discrete stability analysis shows instability even though the semidiscrete scheme was stable. These results motivate the stability analysis of the fully discrete scheme. We perform the same analysis in the two cases of two and three terms, see Figures 5a and 5b.

We recover similar results as for the semidiscrete case. In the end, the stability analysis shows that CCST6 scheme with third-order Runge-Kutta requires at least three leading terms in the simplified inverse Lax-Wendroff boundary condition to preserve stability. An important result which came out of this stability study is that central compact schemes with third-order Runge-Kutta provided with the SILW procedure for the inflow boundary will be stable under the same CFL as that for the periodic boundary case or will not be stable for any CFL, depending on the number of leading terms used. This would not be the case of finite difference schemes of odd order of accuracy. In this last case, one can find that using the minimum number of leading terms required by SILW inflow boundary to maintain the stability decreases the CFL condition. Adding one 


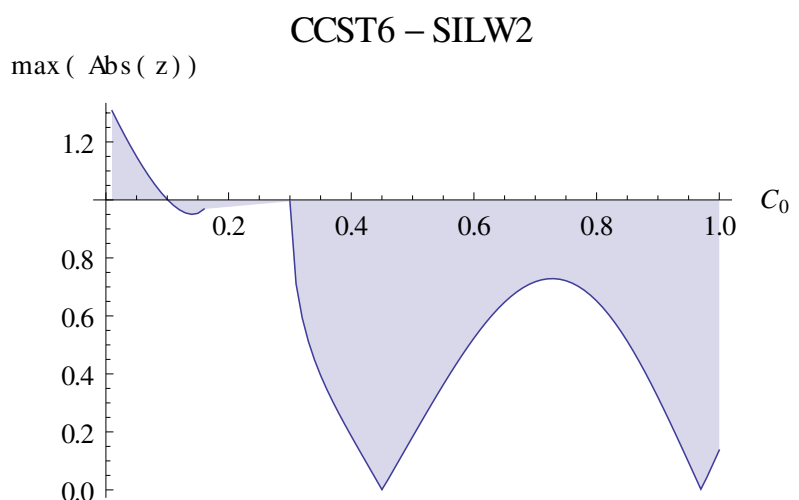

(a) Two leading terms.

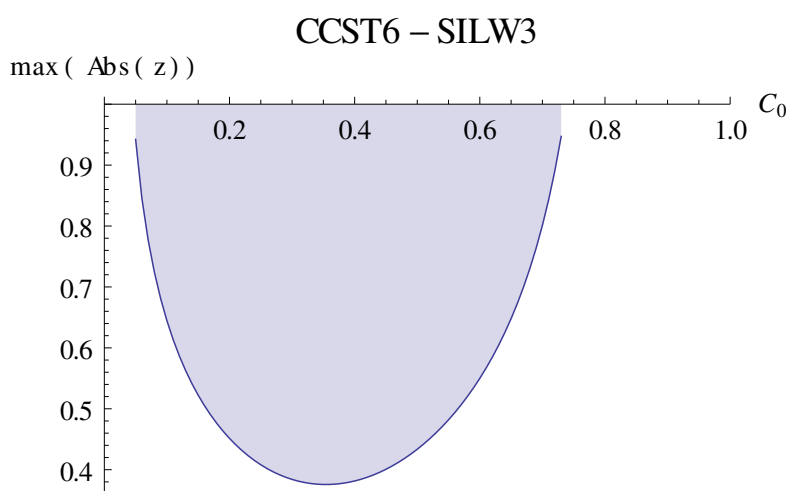

(b) Three leading terms.

FIGURE 5. Maximum of the absolute value of the eigenvalues as function of $C_{0}$ for the RK3CCST6 scheme with the SILW boundary condition with two and three leading terms, and $\lambda_{c f l}=0.961$.

more term in the procedure and one recovers the periodic boundary case CFL condition. For example, for the fifth order finite difference scheme with third-order TVD Runge-Kutta, two leading terms is enough in the SILW boundary to maintain stability. However, doing so the maximum CFL number decreases from 1.43 to 1.02. Using three leading terms allows us to use 1.43 , the same maximum CFL condition as for the periodic case.

The stability analysis presented here has been performed, first in the semidiscrete case and then in the fully discrete one, on different central compact schemes. The starting point of this procedure is the use of normal mode analysis and G-K-S theory. This powerful technique allows us to analytically prove stability of schemes on a finite-domain, independently of the mesh resolution. The stability is then broken into the summation of three simpler problems. However, this technique has some disadvantages. The theory used is rather complex. And the algebra involved can be extremely heavy and not practical for very high orders of accuracy, even using a mathematical software. These drawbacks have motivated us to study stability in a different manner, visualizing the eigenvalue spectrum of the discretized operator. It would not be this time an analytical demonstration of stability, but it gives an apprehension of the behavior of the studied methods. Knowing that no instability comes from the outflow extrapolation boundary condition, we will see that the results obtained by the spectrum analysis are perfectly consistent with the ones obtained in the study of the inflow quarter-plane problem presented in this section.

\subsection{Eigenvalue spectrum visualization}

To confirm and extend to very high order of accuracy the stability analysis done in the previous sections, an eigenspectrum study is done hereon. The analysis begins by considering the one-dimensional linear initial boundary value problem

$$
\begin{cases}\frac{\partial u}{\partial t}+A \frac{\partial u}{\partial x}=0, & x \in\left[x_{A}, x_{B}\right], t \geq 0, \\ u\left(x_{A}, t\right)=g(t), & t \geq 0, \\ u(x, 0)=u_{0}(x), & x \in\left[x_{A}, x_{B}\right],\end{cases}
$$

where $A>0$ is assumed. The left boundary is then an inflow boundary and the right an outflow boundary. The prescribed condition $g(t)$ can again be set to zero without loss of generality. The domain is then discretized 
into $n$ equal intervals of size $\Delta x$, such as $x_{A} \leq x_{0}<x_{1}<\ldots<x_{n} \leq x_{B}$. Notice that both $x_{0}$ and $x_{n}$ are not necessarily located on the boundaries. Practically, we have

$$
x_{A}=x_{0}-C_{A} \Delta x \quad \text { and } \quad x_{A}=x_{n}+C_{B} \Delta x,
$$

where $C_{A}$ and $C_{B}$ are defined respectively in $(0,1]$ and in $[0,1)$.

Remark 4.4. In the particular case where $C_{A}=0$, the number of unknowns is decreased by one, node $x_{0}$ corresponding to the boundary $x_{A}$. Also, for $C_{B}=1$, the number of unknowns is increased by one, ghost point $x_{n+1}$ corresponding to the boundary $x_{b}$.

In order to discretize the considered problem (4.15), central compact schemes are used for the inner points. The ghost points located beyond the outflow boundary are evaluated by an extrapolation procedure, and the ones located to the left of the inflow boundary are determined either by the ILW procedure or its simplified version SILW. Finally, the semidiscrete scheme yields a linear system of equations expressed in a matrix-vector form as

$$
\mathrm{P} \frac{\mathrm{d} \boldsymbol{U}}{\mathrm{d} t}=-\frac{A}{\Delta x} \mathrm{Q} \boldsymbol{U}
$$

where the matrix $\mathrm{P}$ is invertible, and $\boldsymbol{U}$ is the vector representing the values of the solution at the nodes, $\boldsymbol{U}=\left(u_{0}, u_{1}, \ldots, u_{n}\right)^{\mathrm{t}}$. This system contains the chosen inner scheme as well as the two boundary treatments. As before, we first study the considered problem in its semidiscrete form.

\subsubsection{Semidiscrete case}

The normal mode analysis is applied to the matrix-vector system of equations to get the eigenvalue problem. Assuming a solution of the form $u(x, t)=\mathrm{e}^{s t} u_{0}(x)$, the semidiscrete scheme yields

$$
\widetilde{s} \mathrm{P} \boldsymbol{U}=-\operatorname{sgn}(A) \mathrm{Q} \boldsymbol{U},
$$

with $\widetilde{s}=s \frac{\Delta x}{|A|}$ being the considered eigenvalue. The next step is to compute the eigenvalues of the matrix $-\operatorname{sgn}(A) \mathrm{P}^{-1} \mathrm{Q}$. In the practical application, the choice made on the velocity has been $A=1$, for the sake of simplicity. As before, the semidiscretization provided with the considered boundary conditions, on the studied mesh, is stable if the whole eigenvalue spectrum lies in the left half-plane $(\operatorname{Re}(\widetilde{s}) \leq 0)$, as in Figure 6 .

The results displayed in Figure 6 correspond to the case of outflow extrapolation and inflow inverse Lax-Wendroff boundaries, on a grid made of 40 cells. It is clear that for all central compact schemes derived from the general formula (2.6), these particular boundary treatments maintain stability, due to the fact that the spectra lie in the left half-plan. Similar results are obtained with different grid resolutions. This result is perfectly consistent with the one obtained using the G-K-S theory because it has been proved previously that these two boundary conditions are stable separately. However, we know that the SILW procedure stability depends on the position of the boundary and on the number of leading terms used. The same example of the CCS-T6 scheme is used to exhibit our results.

In Figure 7, the spectra are displayed for different grids, from 20 to 320 cells. One can see that for some positions of the boundary, see Figures $7 \mathrm{a}$ and $7 \mathrm{c}$, some particular eigenvalues are fixed and independent of the mesh resolution. These specific eigenvalues are the ones responsible of instability in cases where their real part is greater than zero. This is why our study will be focused on these fixed eigenvalues. The remainder of the spectra is similar to the ones found with ILW boundary and corresponds to a stable scheme. It appears that these particular eigenvalues can be identified as the ones found with the G-K-S theory for the inflow boundary. That is why, in cases where no function were found to be solution of the characteristic equation and of the boundary schemes, we observe that no such particular eigenvalues are present in the spectra, see Figure $7 \mathrm{~b}$. To ensure that both stability studies lead to the same results, we locate these particular eigenvalues in the spectra, and then plot their real part depending upon the inflow boundary position $C_{A}$, see Figure 8b. 


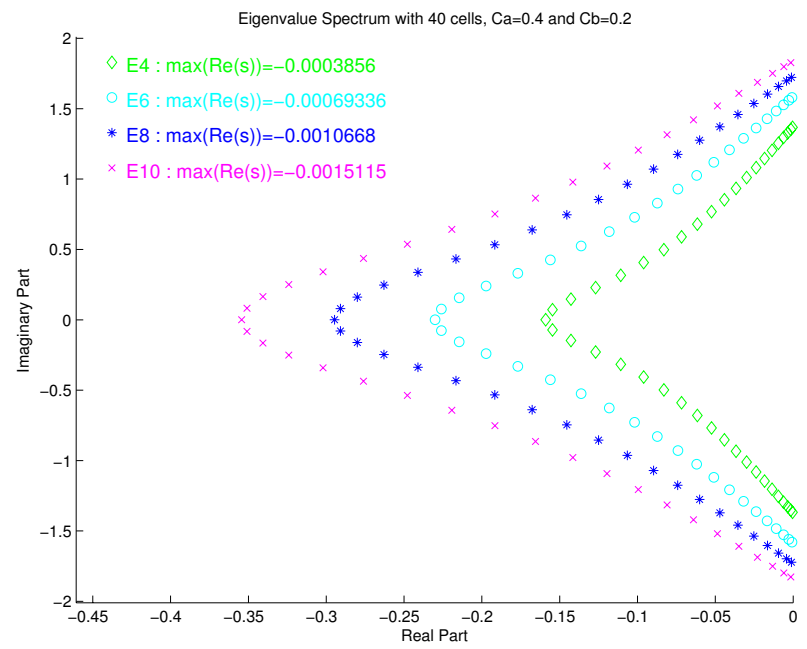

(a) CCS-E.

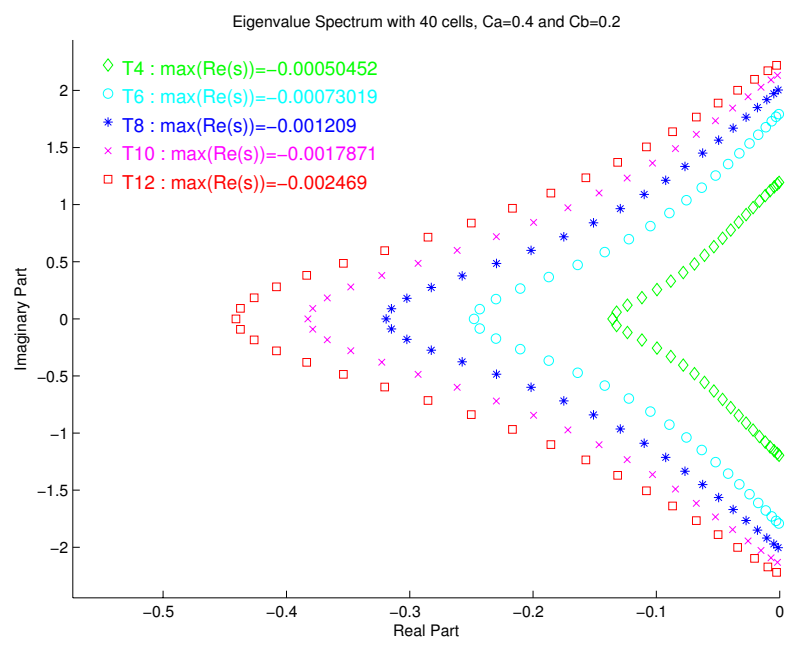

(b) CCS-T.

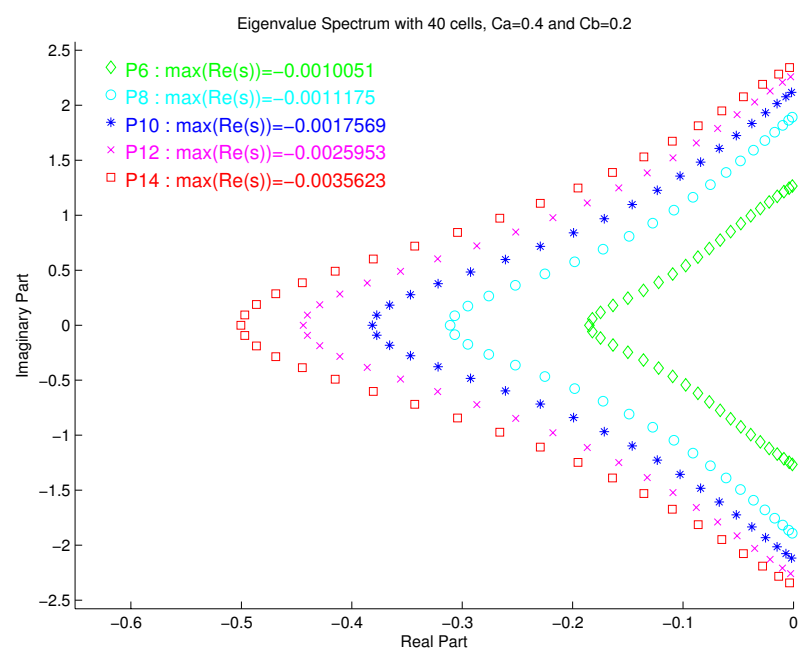

(c) CCS-P.

FigURE 6. The eigenvalue spectra of semi-discrete central compact schemes, closed with an inverse Lax-Wendroff procedure for the inflow boundary, and extrapolation for the outflow boundary, with 40 cells, $C_{A}=0.4$ and $C_{B}=0.2$.

It is clear in Figure 8 these two different stability analysis lead to exactly the same results. Needless to say that eigenspectrum analysis gives not an analytical proof of stability, being dependent on the mesh resolution. But considering the fact that instability comes from particular eigenvalues independent of the grid, we obtain equivalent results. Performing the same comparison in the cases of two and three leading terms in the SILW boundary treatment leads to the same consistency in the results, see Figure 9. Going to four terms, one would observe that these particular eigenvalues vanish, and thus one recovers a stable spectrum similar to the ILW case. Let us now look at the fully discrete case. 


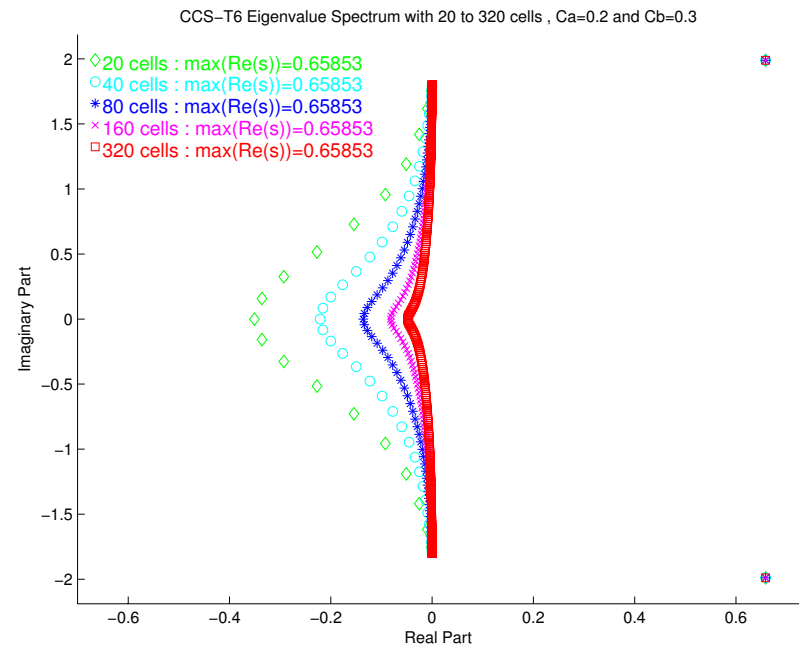

(a) $C_{A}=0.2$.

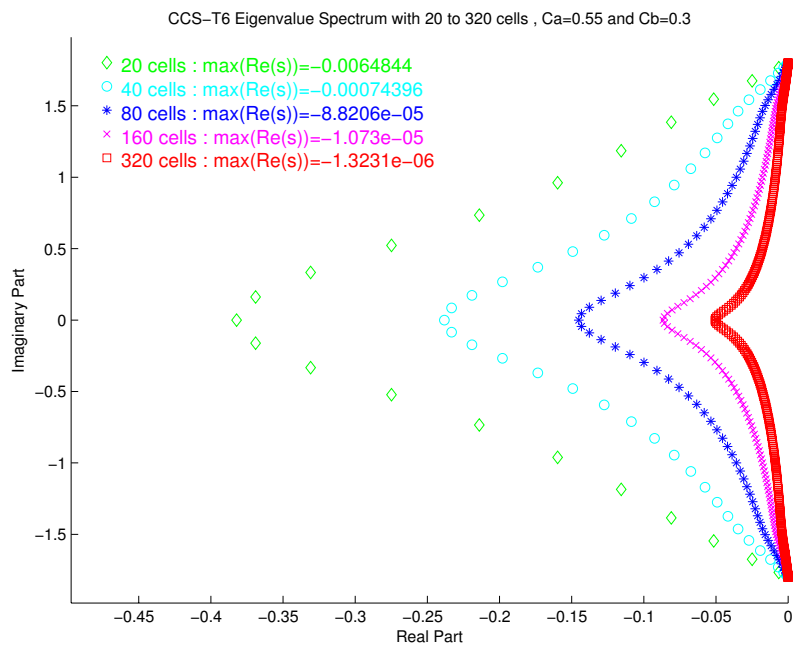

(b) $C_{A}=0.55$.

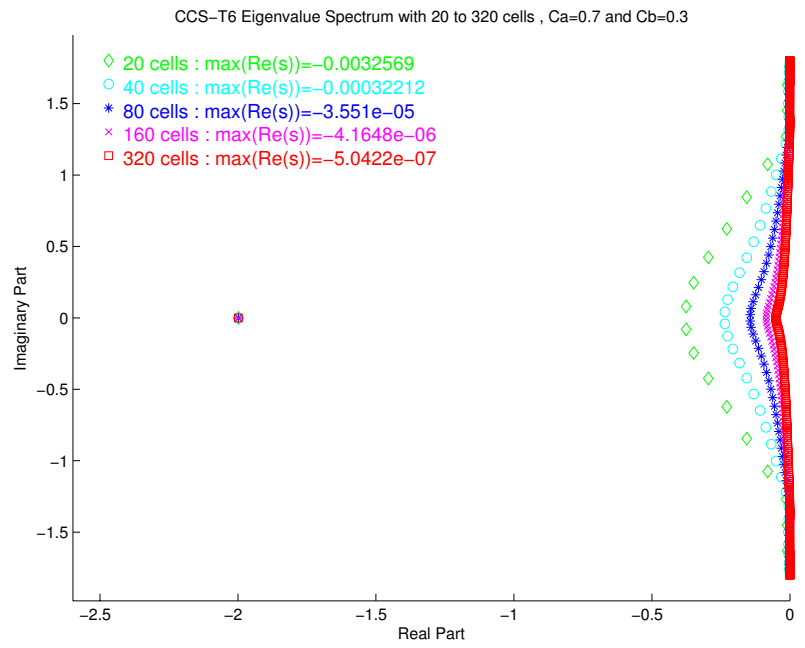

(c) $C_{A}=0.7$.

Figure 7. The eigenvalue spectra of the CCS-T6, provided with SILW procedure with one term for the inflow boundary, and extrapolation for the outflow boundary.

\subsubsection{Fully discrete case}

Here, we recall the semi-discrete system (4.16) obtained previously

$$
\mathrm{P} \frac{\mathrm{d} \boldsymbol{U}}{\mathrm{d} t}=-\frac{A}{\Delta x} \mathrm{Q} \boldsymbol{U}
$$

We apply now to this ODE the third-order TVD Runge-Kutta method. Doing so, the fully discretize problem can be written as

$$
\boldsymbol{U}^{n+1}=\mathrm{G} \boldsymbol{U}^{n}
$$




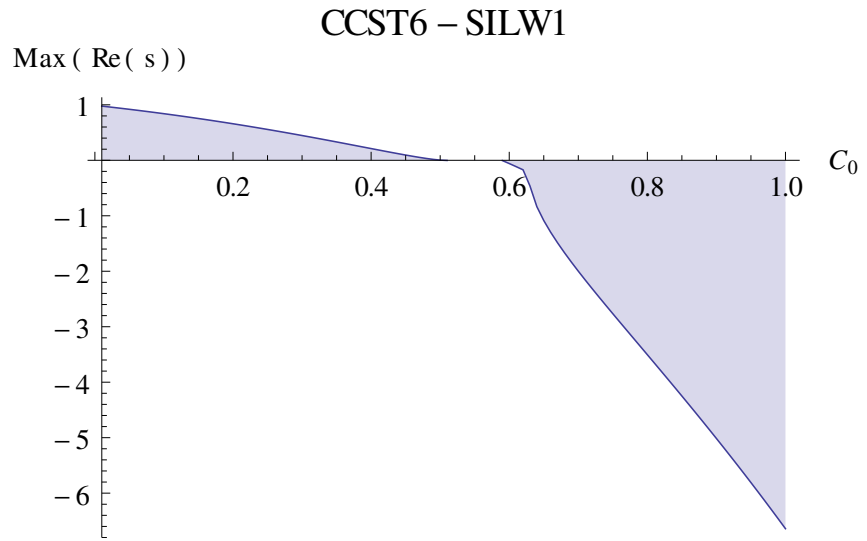

(a) G-K-S analysis.
Particular eigenvalue real part of CCST6-SILW1

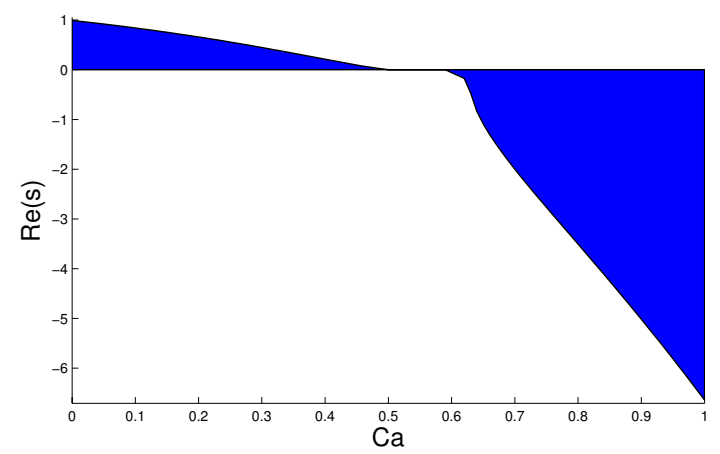

(b) Eigenspectrum analysis.

FiguRE 8. Real part of the CCS-T6 scheme particular eigenvalues, provided with extrapolation and SILW with one term boundary conditions.

where $\boldsymbol{U}^{n}$ and $\boldsymbol{U}^{n+1}$ are the solution vectors respectively at time $t^{n}$ and $t^{n+1}$. In equation (4.18), the fully discrete operator $\mathrm{G}$ is defined as

$$
\mathrm{G}=\mathrm{I}_{d}-\operatorname{sgn}(A) \frac{|A| \Delta t}{\Delta x} \mathrm{P}^{-1} \mathrm{Q}+\left(\frac{|A| \Delta t}{\Delta x}\right)^{2}\left(\mathrm{P}^{-1} \mathrm{Q}\right)^{2}-\operatorname{sgn}(A)\left(\frac{|A| \Delta t}{\Delta x}\right)^{3}\left(\mathrm{P}^{-1} \mathrm{Q}\right)^{3}
$$

where $I_{d}$ is the identity matrix.

Making use again of the normal mode analysis to derive the eigenvalue problem, we assume a solution of the form $u^{n}=z^{n} u^{0}$. Substituting this solution in (4.18), one gets

$$
z \boldsymbol{U}^{n}=\mathrm{G}\left(\frac{|A| \Delta t}{\Delta x}\right) \boldsymbol{U}^{n} .
$$

To ensure stability of the fully discrete problem we compute the complex eigenvalues of the operator G, which depends on $\frac{|A| \Delta t}{\Delta x}$. We make use here of the same CFL definition as before, $\lambda_{c f l}=\frac{|A| \Delta t}{\Delta x}$. Consistent with the G-K-S analysis performed previously, the eigenspectrum computation is done at first using the same CFL condition as that for the periodic boundary case, and then using a decreasing sequence, re-performing at each step the stability analysis. In the end, the scheme will be stable if the whole eigenvalue spectrum lies in the unit circle, i.e. $|z| \leq 1$, as in Figure 10 .

In Figure 10, the results correspond to the case of outflow extrapolation and inflow inverse Lax-Wendroff boundaries, on a grid made of 80 cells. It shows that for all central compact schemes, these particular boundary treatments maintain stability due to the fact that the spectra lie in the unit circle. Similar results are obtained with different grid resolutions. Let us look at the stability behavior of the simplified inverse Lax-Wendroff procedure, in the same case of the CCS-T6 scheme.

In a similar way as before, we observe in Figures 11a and 11c the presence of particular eigenvalues independent of the number of cells, for some boundary positions. Again, because these are the ones responsible for the instabilities, we focus our study on these by locating them and plotting their absolute values depending on the inflow boundary position $C_{A}$, see Figure 12 .

In the end, this eigenvalue spectrum analysis leads to the same results as before. The outflow extrapolation and inflow inverse Lax-Wendroff boundary treatments maintain the stability, under the same CFL condition 


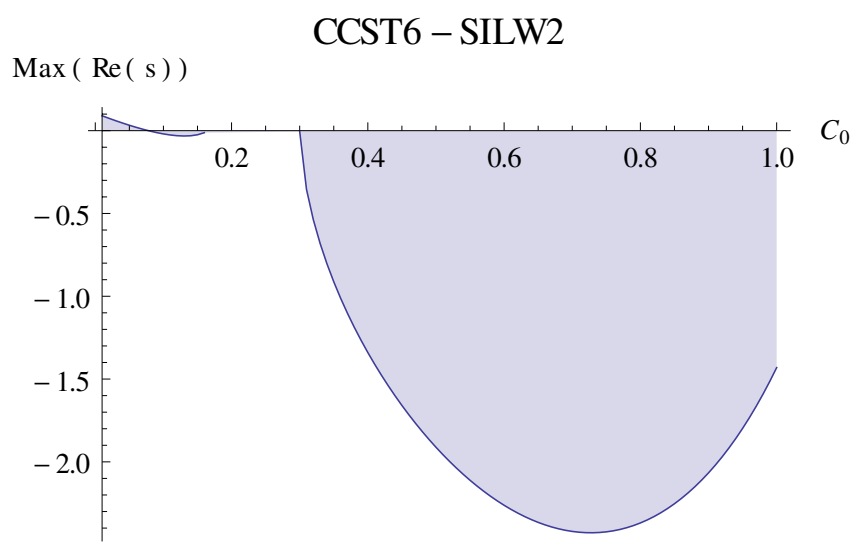

(a) G-K-S analysis.

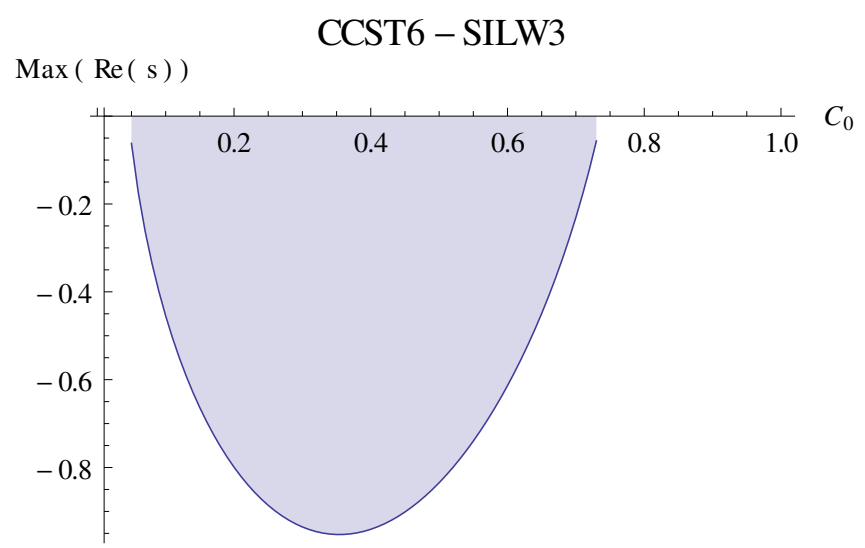

(c) G-K-S analysis.
Particular eigenvalue real part of CCST6-SILW2

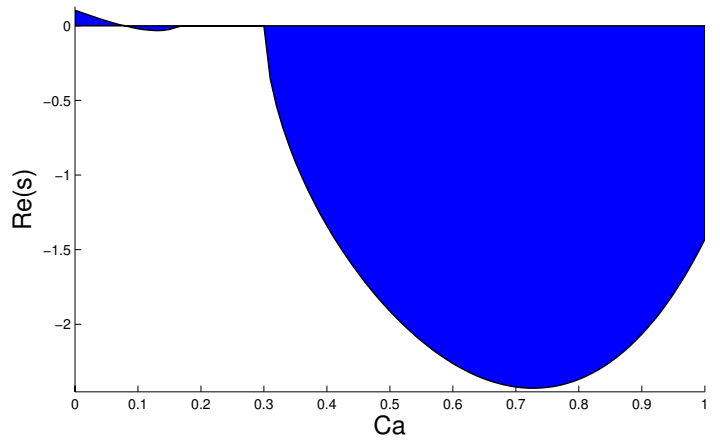

(b) Eigenspectrum analysis.

Particular eigenvalue real part of CCST6-SILW3

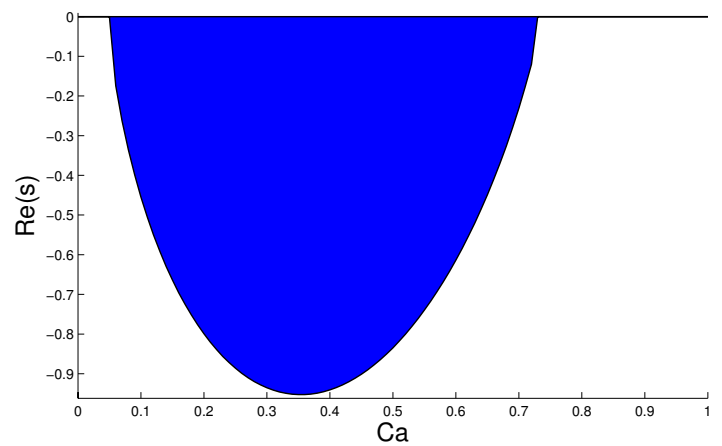

(d) Eigenspectrum analysis.

Figure 9. Real part of CCS-T6 scheme particular eigenvalues, provided with extrapolation and SILW with two and three terms boundary conditions.

as that for the periodic boundary case, for any central compact schemes presented and for any position of the boundary. The simplified inverse Lax-Wendroff boundary stability depends on the number of leading terms $k_{d}$ used in the procedure. Nevertheless, SILW maintains stability of central compact schemes under the same CFL as the periodic case if enough terms are used, or is not stable for any CFL condition.

Finally, in Table 1, we gather the results of the stability analysis, giving the minimum number of leading terms required in the SILW inflow boundary treatment for the central compact schemes to remain stable, using the third-order TVD Runge-Kutta as time discretization, under the CFL conditions gathered in Table 2 ensuring the inner scheme to be Cauchy stable.

To attest the relevance of the stability analysis presented, we give a numerical example. We consider the linear advection case (4.15) with $A=1$, on the domain $[0,1]$. The initial solution writes $u_{0}(x)=0.25+0.5 \sin (\pi x)$, while the inflow boundary condition is given by $u(-1, t)=0.25-0.5 \sin (\pi(1+t))$. The spatial discretization comes from the use of the sixth order tridiagonal central compact scheme (CCS-T6) with third-order TVD Runge-Kutta time discretization. Regarding the boundary treatments the ghost point values are evaluated with an extrapolation at the outflow boundary, and through the use of the simplified inverse Lax-Wendroff 
RK3-CCSE Discrete Eigenvalue Spectrum with 80 cells , $\mathrm{Ca}=0.3$ and $\mathrm{Cb}=0.3$ RK3-CCST Discrete Eigenvalue Spectrum with 80 cells , $\mathrm{Ca}=0.3$ and $\mathrm{Cb}=0.3$

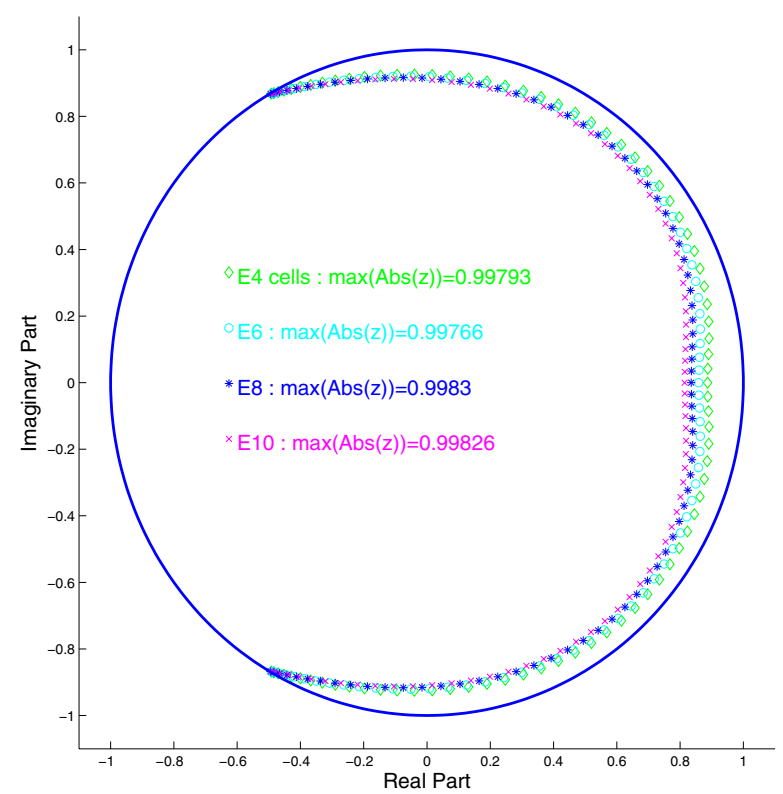

(a) CCS-E.

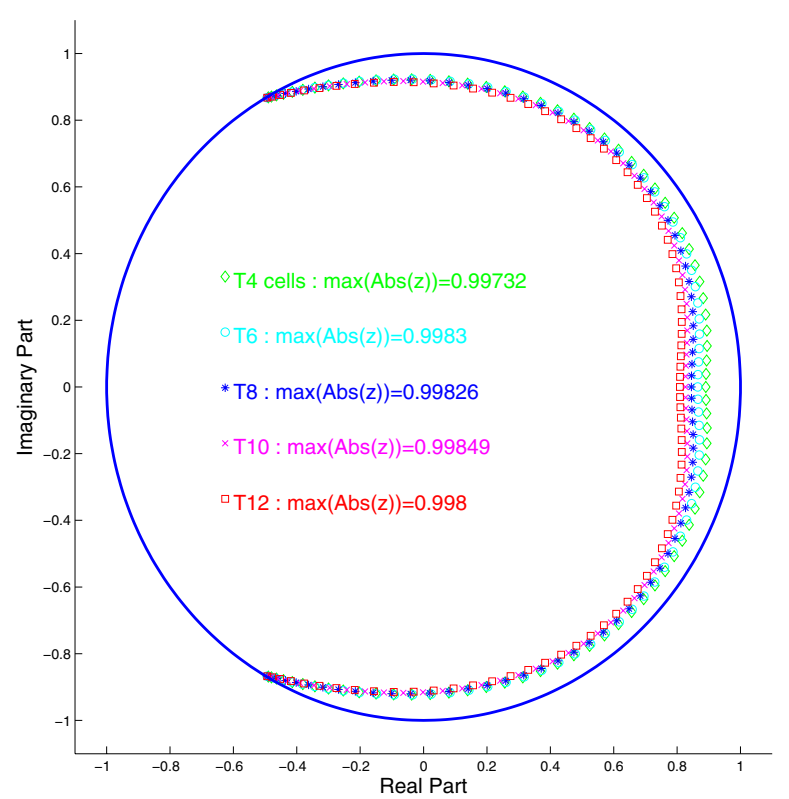

(b) CCS-T.

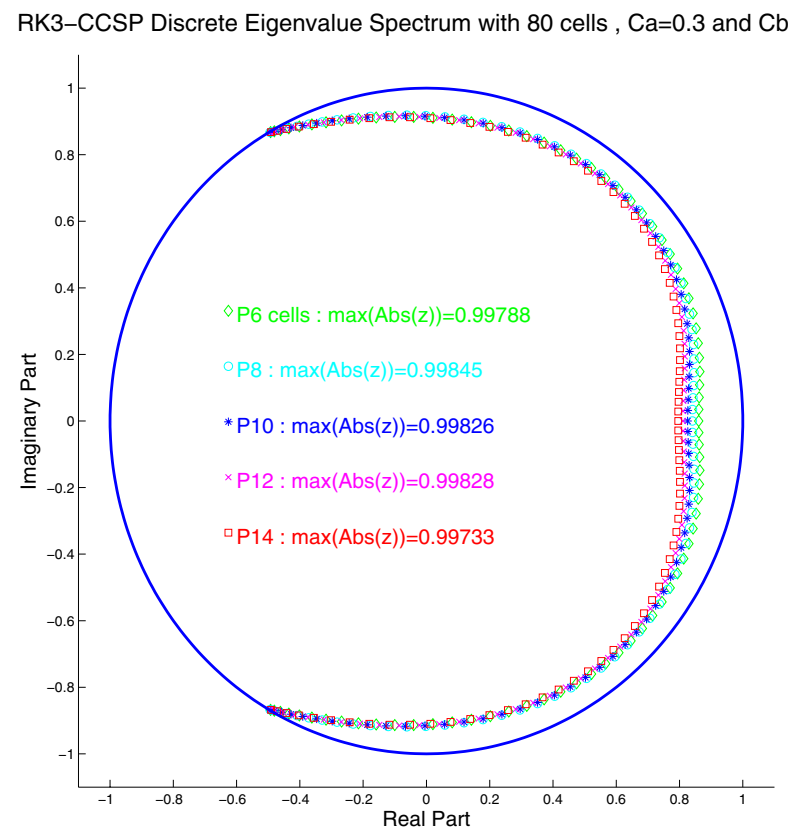

(c) CCS-P.

Figure 10. The eigenvalue spectra of the RK3-CCS, closed with an inverse Lax-Wendroff procedure for the inflow boundary, and extrapolation for the outflow boundary with 80 cells, $C_{A}=0.3$ and $C_{B}=0.3$, and the maximum CFL condition ensuring inner schemes stability. 
RK3-CCST6 Discrete Eigenvalue Spectrum with 40 to 320 cells, $\mathrm{Ca}=0.2$ and $\mathrm{Cb}=0.3 \mathrm{RK} 3-\mathrm{CCST} 6$ Discrete Eigenvalue Spectrum with 40 to 320 cells, Ca=0.55 and $\mathrm{Cb}=0.3$

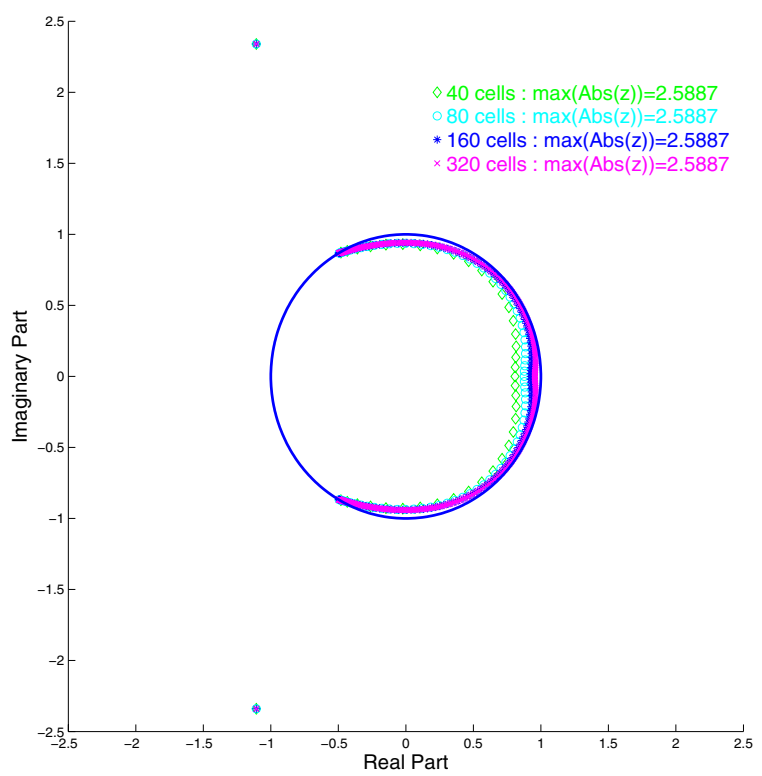

(a) $C_{A}=0.2$.

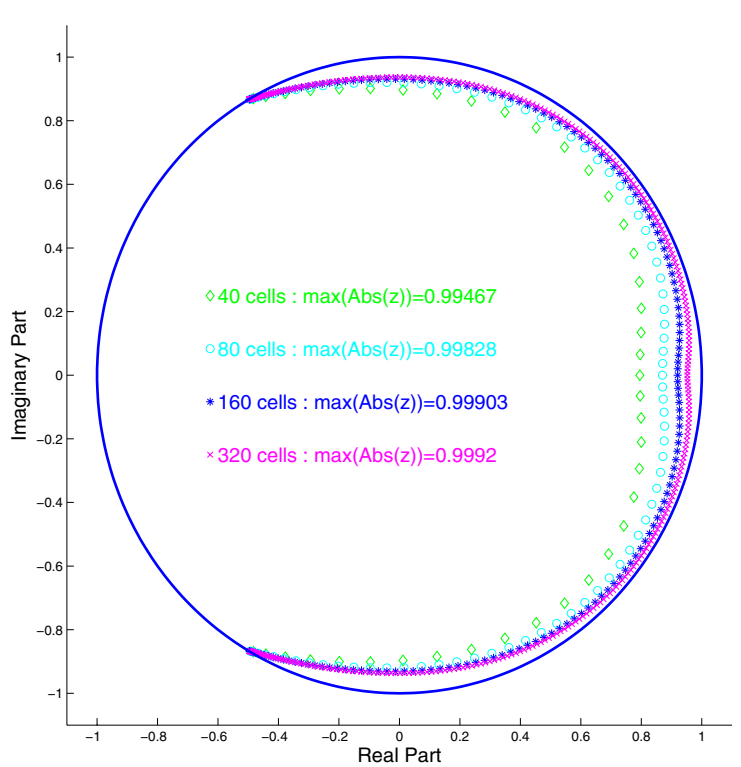

(b) $C_{A}=0.55$.

RK3-CCST6 Discrete Eigenvalue Spectrum with 40 to 320 cells, $\mathrm{Ca}=0.7$ and $\mathrm{Cb}=0.3$

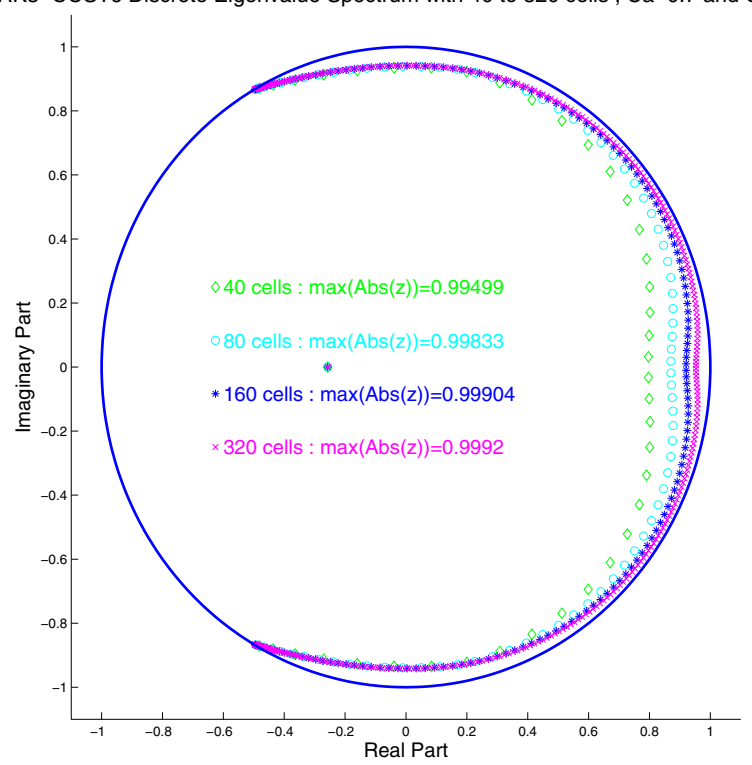

(c) $C_{A}=0.7$.

Figure 11. The eigenvalue spectra of the RK3-CCST6, provided with SILW procedure with one term for the inflow boundary, and extrapolation for the outflow boundary, and $\lambda_{c f l}=0.961$. 


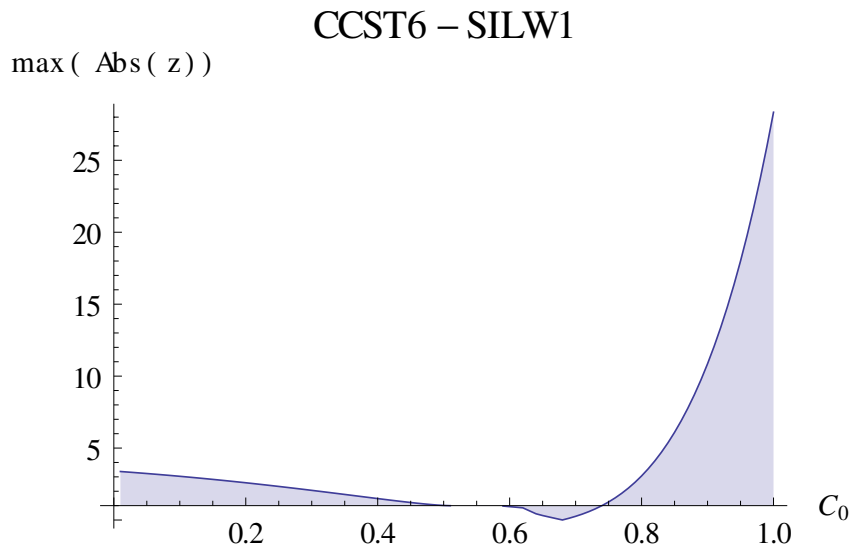

(a) G-K-S analysis.
Particular eigenvalue absolute value of CCST6-SILW1

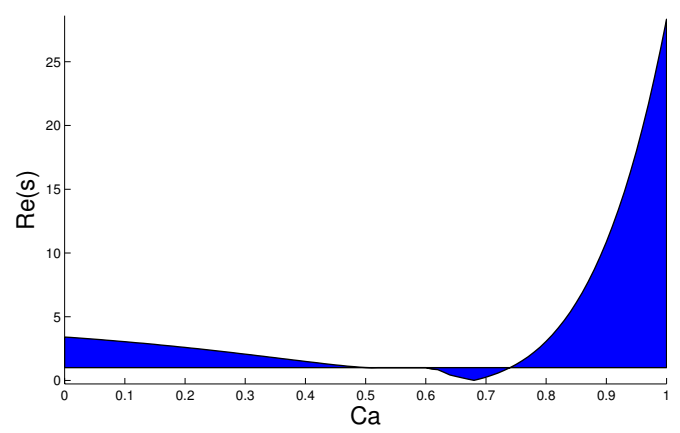

(b) Eigenspectrum analysis.

FiguRE 12. Absolute value of the particular eigenvalues of RK3-CCST6 scheme provided with extrapolation and SILW with one term boundary conditions, and $\lambda_{c f l}=0.961$.

TABLE 1. Minimum number of leading terms required by the different RK3-CCS schemes to remain stable under the same CFL as that for periodic boundary conditions.

\begin{tabular}{lc}
\hline Scheme & Required leading terms \\
\hline CCS-E4 & 3 \\
CCS-E6 & 4 \\
CCS-E8 & 5 \\
CCS-E10 & 5 \\
\hline
\end{tabular}

\begin{tabular}{lc}
\hline Scheme & Required leading terms \\
\hline CCS-T4 & 3 \\
CCS-T6 & 3 \\
CCS-T8 & 5 \\
CCS-T10 & 8 \\
CCS-T12 & 9 \\
\hline
\end{tabular}

\begin{tabular}{lc}
\hline Scheme & Required leading terms \\
\hline CCS-P6 & 4 \\
CCS-P8 & 5 \\
CCS-P10 & 7 \\
CCS-P12 & 9 \\
CCS-P14 & 9 \\
\hline
\end{tabular}

procedure at the inflow boundary. We consider the case where $C_{A}=0.001$ and $C_{B}=0.7$, and thus the first and last grid points are located as

$$
x_{0}=0.001 \Delta x \quad \text { and } \quad x_{n}=1-0.7 \Delta x .
$$

A 40 cells uniform grid is used. We clearly see in Figure 14 that the outflow boundary $x_{B}=1$ and the last grid point $x_{n}$ do not coincide. On the other hand, at $x_{A}=0$ it is impossible to distinguish the two points, the boundary and the first grid point $x_{0}$ being extremely close.

Thanks to the stability analysis presented, one knows that using the CCS-T6 scheme and in the case where the first grid point is too close to the boundary, two leading terms in the SILW boundary treatment is not enough to maintain stability. On Figure 14a, we clearly observe this unstable behavior. The stability analysis tells us that three terms in the SILW procedure can enforce stability for any position of the boundary. The results displayed in Figure 14b confirm this statement. Furthermore, after a very long computation time $t=10000$, 


$$
\max (\operatorname{Abs}(\mathrm{z}))
$$$$
\text { CCST6 - SILW2 }
$$

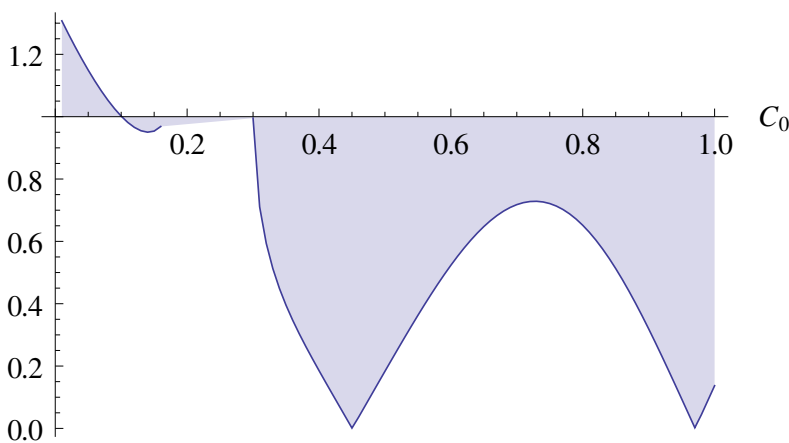

(a) G-K-S analysis.

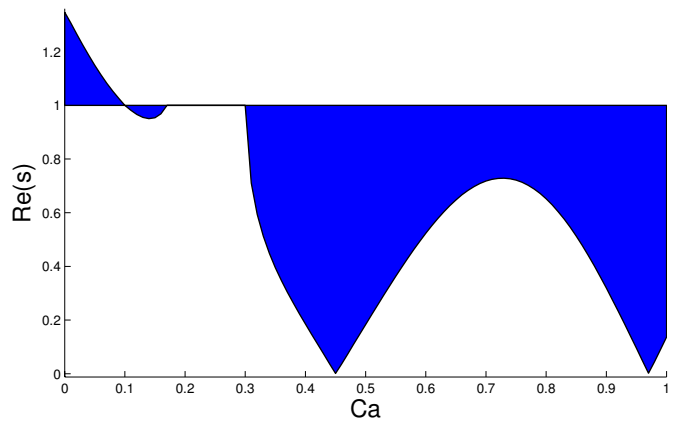

(b) Eigenspectrum analysis.

Particular eigenvalue absolute value of CCST6-SILW3

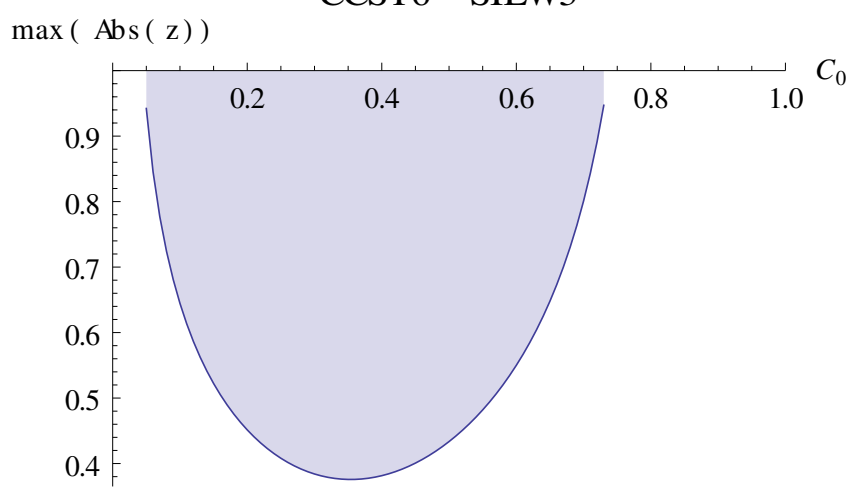

(c) G-K-S analysis.

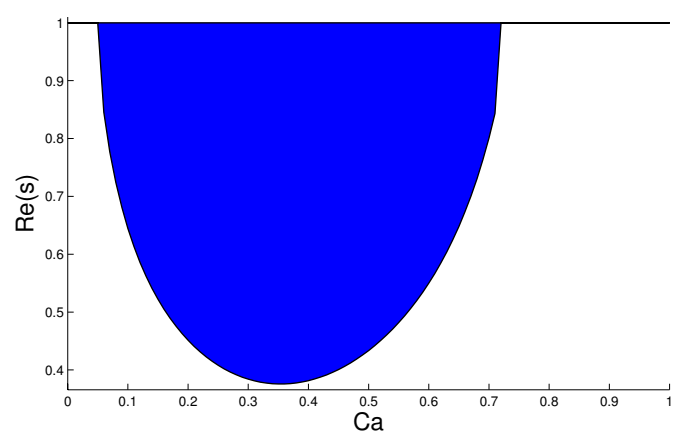

(d) Eigenspectrum analysis.

Figure 13. Absolute value of the particular eigenvalues of RK3-CCST6 scheme provided with extrapolation and SILW with two and three terms boundary conditions, and $\lambda_{c f l}=0.961$.

TABLE 2. Maximum CFL, $\max \left(\lambda_{c f l}\right)$, ensuring inner RK3-CCS schemes to be Cauchy stable.

\begin{tabular}{|c|c|c|c|}
\hline & & \multirow[b]{2}{*}{ Maximum CFL } & \\
\hline & Scheme & & \\
\hline & CCS-E4 & 1.26 & \\
\hline & CCS-E6 & 1.09 & \\
\hline & CCS-E8 & 1.00 & \\
\hline & CCS-E10 & 0.942 & \\
\hline Scheme & Maximum CFL & Scheme & Maximum CFL \\
\hline CCS-T4 & 1.44 & CCS-P6 & 1.36 \\
\hline CCS-T6 & 0.961 & CCS-P8 & 0.911 \\
\hline CCS-T8 & 0.859 & CCS-P10 & 0.813 \\
\hline CCS-T10 & 0.807 & CCS-P12 & 0.761 \\
\hline CCS-T12 & 0.774 & CCS-P14 & 0.732 \\
\hline
\end{tabular}




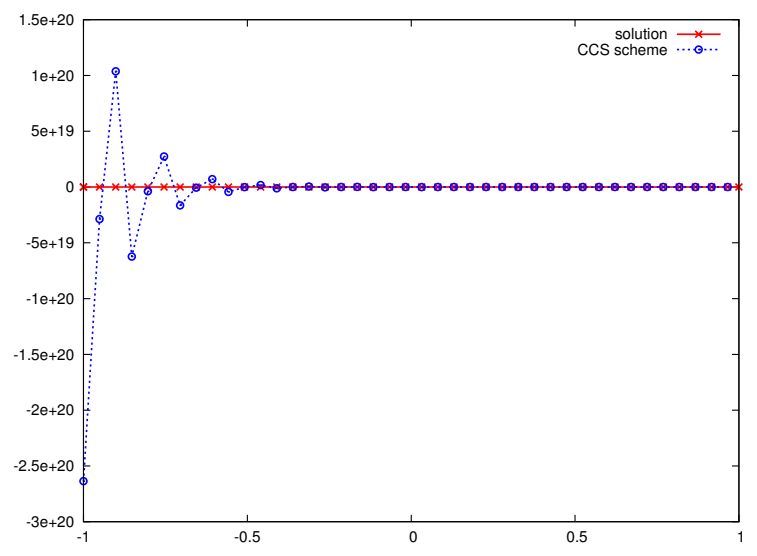

(a) Two leading terms, $t=10$.

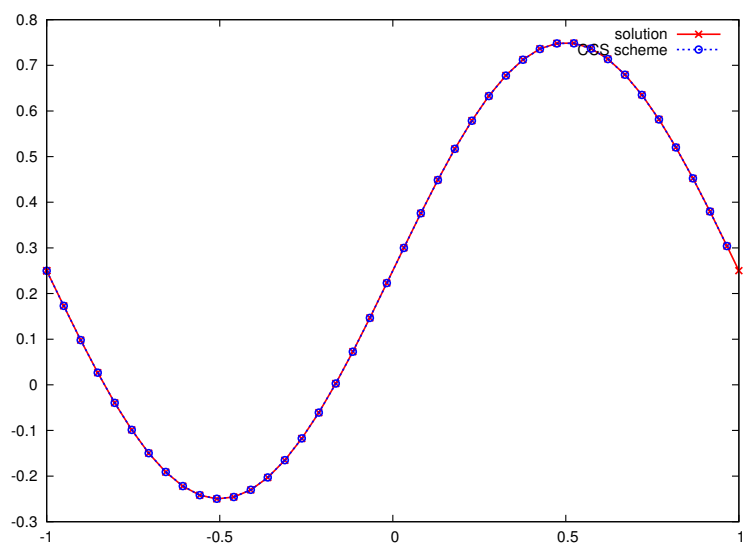

(b) Three leading terms, $t=10000$.

FiguRE 14. Numerical results obtained with RK3-CCST6 scheme provided with extrapolation and SILW boundaries in the linear advection case $(A=1)$ on $x \in[-1,1]$, with the initial and boundary condition $u_{0}(x)=0.25+0.5 \sin (\pi x)$ and $u(-1, t)=0.25-0.5 \sin (\pi(1+t))$, with 40 cells, $C_{A}=0.001$ and $\lambda_{c f l}=0.961$.

TABLE 3. Rate of convergence of the RK3-CCST6 scheme provided with outflow extrapolation and inflow SILW with three leading terms in the two cases of linear advection and Burgers equation, with $C_{A}=0.001$ and $C_{B}=0.7$. (a) linear advection. (b) Burgers equation.

\begin{tabular}{ccccc}
\hline \multicolumn{3}{c}{$L_{2}$} & \multicolumn{2}{c}{$L_{\infty}$} \\
\hline$h$ & $E_{L_{2}}^{h}$ & $q_{L_{2}}^{h}$ & $E_{L_{\infty}}^{h}$ & $q_{L_{\infty}}^{h}$ \\
\hline$\frac{1}{20}$ & $8.79 \mathrm{E}-5$ & 5.98 & $1.04 \mathrm{E}-4$ & 5.99 \\
$\frac{1}{40}$ & $1.55 \mathrm{E}-6$ & 5.99 & $1.81 \mathrm{E}-6$ & 5.98 \\
$\frac{1}{80}$ & $2.56 \mathrm{E}-8$ & 6.00 & $3.03 \mathrm{E}-8$ & 5.99 \\
$\frac{1}{160}$ & $4.10 \mathrm{E}-10$ & 6.00 & $4.88 \mathrm{E}-10$ & 6.00 \\
$\frac{1}{320}$ & $6.48 \mathrm{E}-12$ & - & $7.74 \mathrm{E}-12$ & - \\
\hline
\end{tabular}

\begin{tabular}{ccccc}
\hline \multicolumn{3}{c}{$L_{2}$} & \multicolumn{2}{c}{$L_{\infty}$} \\
\hline$h$ & $E_{L_{2}}^{h}$ & $q_{L_{2}}^{h}$ & $E_{L_{\infty}}^{h}$ & $q_{L_{\infty}}^{h}$ \\
\hline$\frac{1}{20}$ & $2.26 \mathrm{E}-5$ & 5.23 & $5.06 \mathrm{E}-5$ & 4.50 \\
$\frac{1}{40}$ & $6.20 \mathrm{E}-7$ & 6.27 & $2.41 \mathrm{E}-6$ & 5.85 \\
$\frac{1}{80}$ & $8.51 \mathrm{E}-9$ & 6.51 & $4.41 \mathrm{E}-8$ & 6.33 \\
$\frac{1}{160}$ & $9.58 \mathrm{E}-11$ & 6.28 & $5.63 \mathrm{E}-10$ & 6.33 \\
$\frac{1}{320}$ & $1.25 \mathrm{E}-12$ & - & $7.09 \mathrm{E}-12$ & - \\
\hline
\end{tabular}

no dissipation or dispersion errors can be visually observed. This result also confirms the high accuracy of the presented central compact schemes provided with the high order boundary treatments studied.

At last, to ensure the studied boundary treatments preserve the high accuracy of the schemes, we compute the rate of convergence of the RK3-CCST6 scheme provided with extrapolation and SILW with three leading terms boundary treatments, in the same linear advection case than before, for two different inflow boundary positions, $C_{A}=0.001$ and $C_{A}=1$, see Tables $3 \mathrm{a}$ and $4 \mathrm{a}$. We also perform this analysis in the Burgers case, $u_{t}+\left(\frac{1}{2} u^{2}\right)_{x}=0$, with the same smooth initial data than for the linear case, see Tables $3 \mathrm{~b}$ and $4 \mathrm{~b}$. In this nonlinear case, we do not give explicitly the prescribed boundary condition and its successive derivatives due to the complexity of the formula. To ensure that the error of the time discretization will not dominate, we adjust the time step to $\Delta t=\Delta x^{d / 3}$, where $d$ is the order of accuracy of the spatial discretization.

The results displayed clearly exhibit that the expected accuracy is obtained numerically.

To end this stability study, a brief discussion on energy stability is addressed. 
TABLE 4. Rate of convergence of the RK3-CCST6 scheme provided with outflow extrapolation and inflow SILW with three leading terms in the two cases of linear advection and Burgers equation, with $C_{A}=1$ and $C_{B}=0.7$. (a) linear advection. (b) Burgers equation.

\begin{tabular}{ccccc}
\hline \multicolumn{3}{c}{$L_{2}$} & \multicolumn{2}{c}{$L_{\infty}$} \\
\hline$h$ & $E_{L_{2}}^{h}$ & $q_{L_{2}}^{h}$ & $E_{L_{\infty}}^{h}$ & $q_{L_{\infty}}^{h}$ \\
$\frac{1}{20}$ & $5.92 \mathrm{E}-5$ & 6.00 & $7.74 \mathrm{E}-5$ & 5.96 \\
$\frac{1}{40}$ & $1.18 \mathrm{E}-6$ & 6.00 & $1.57 \mathrm{E}-6$ & 5.98 \\
$\frac{1}{80}$ & $2.08 \mathrm{E}-8$ & 6.00 & $2.81 \mathrm{E}-8$ & 5.99 \\
$\frac{1}{160}$ & $3.45 \mathrm{E}-10$ & 6.00 & $4.70 \mathrm{E}-10$ & 6.00 \\
$\frac{1}{320}$ & $5.56 \mathrm{E}-12$ & - & $7.60 \mathrm{E}-16$ & - \\
\hline
\end{tabular}

\begin{tabular}{ccccc}
\hline \multicolumn{3}{c}{$L_{2}$} & \multicolumn{2}{c}{$L_{\infty}$} \\
\hline$h$ & $E_{L_{2}}^{h}$ & $q_{L_{2}}^{h}$ & $E_{L_{\infty}}^{h}$ & $q_{L_{\infty}}^{h}$ \\
\hline$\frac{1}{20}$ & $1.89 \mathrm{E}-5$ & 5.24 & $4.26 \mathrm{E}-5$ & 4.60 \\
$\frac{1}{40}$ & $6.516 \mathrm{E}-7$ & 5.34 & $2.12 \mathrm{E}-6$ & 5.87 \\
$\frac{1}{80}$ & $8.65 \mathrm{E}-9$ & 6.60 & $4.08 \mathrm{E}-8$ & 6.33 \\
$\frac{1}{160}$ & $9.56 \mathrm{E}-11$ & 6.35 & $5.41 \mathrm{E}-10$ & 6.33 \\
$\frac{1}{320}$ & $1.20 \mathrm{E}-12$ & - & $6.95 \mathrm{E}-12$ & - \\
\hline
\end{tabular}

\subsection{Energy stability}

In the previous Section 4.1, the G-K-S theory has demonstrated its high capability and wide application to general initial boundary value problems. However, its high complexity has also been highlighted. Another approach to study stability is the energy method, which has a simple structure and is convenient to use when it works. This method consists in constructing a norm which does not grow from one time level to the next, if the boundary data $g(t)$ is set to zero. The drawback is that if one fails to construct such a norm, it is not clear whether or not the studied scheme is stable or not. Indeed, the energy method only provides a sufficient condition of stability, while G-K-S theory provides necessary and sufficient conditions for stability. Due to its simple structure and also to compete with Galerkin finite element methods and their natural energy property, the issue of energy formulation for difference schemes has been a central problem under study for decades, and has played a crucial role in the characterization of well-posedness in PDEs and stability in numerical schemes. Consequently, the energy stability issue as well as the establishment of a summation-by-part have been tackled in numerous seminal papers and will not be detailed here. The interested reader may refer to $[2,7,8,10,11,16,17,19,21]$. In the remainder, only the main points related to an energy formulation are given.

Let us consider the scalar linear hyperbolic equation

$$
\frac{\partial u}{\partial t}+A \frac{\partial u}{\partial x}=0, \quad 0 \leq x \leq 1, \quad t>0 .
$$

The solution of (4.21) is approximated using compact schemes in the following form

$$
\mathrm{P} \frac{\mathrm{d} \boldsymbol{U}}{\mathrm{d} t}=-\frac{A}{\Delta x} \mathrm{Q} \boldsymbol{U}
$$

where $\boldsymbol{U}=\left(u_{0}, u_{1}, \ldots, u_{n}\right)^{\mathrm{t}}$ is the solution vector and $\left\{x_{j}\right\}_{j=0, \ldots, n}$ are the equally spaced grid points with $x_{0}=0$ and $x_{n}=1$. In (4.22), the operators $\mathrm{P}$ and $\mathrm{Q}$ contain both boundary treatments at $x_{0}$ and $x_{n}$, which remain unspecified for now. Let us introduce the discrete scalar product and norm for real grid vectors $\boldsymbol{U}, \boldsymbol{V} \in \mathbb{R}^{n+1}$ by

$$
\begin{gathered}
(\boldsymbol{U}, \boldsymbol{V})_{h}=(\boldsymbol{U}, \mathrm{HP} \boldsymbol{V})=\Delta x \sum_{i, j=0}^{n}(H P)_{i j} u_{i} v_{j}, \\
\|\boldsymbol{U}\|_{h}^{2}=(\boldsymbol{U}, \boldsymbol{U})_{h},
\end{gathered}
$$

where the matrix $\mathbf{H}=\left(H_{i j}\right)_{i j}$, named here the norm matrix, is defined such that the matrix $\mathrm{HP}=\left(\sum_{k} H_{i k} P_{k j}\right)_{i j}$ is symmetric positive definite. 
Definition 4.5. The difference operator $\mathrm{P}^{-1} \mathrm{Q}$ is semibounded if for all $\boldsymbol{V} \in \mathbb{R}^{n+1}$ the inequality

$$
-\frac{A}{\Delta x}\left(\boldsymbol{V}, \mathrm{P}^{-1} \mathrm{Q} \boldsymbol{V}\right)_{h} \leq \alpha\|\boldsymbol{V}\|_{h}^{2}
$$

holds, where $\alpha$ is a constant independent of $\boldsymbol{V}$ and $\Delta x$.

Using the scalar product and norm defined in (4.23) and (4.24), the following relationship can be easily obtained

$$
\frac{\mathrm{d}}{\mathrm{d} t}\|\boldsymbol{U}\|_{h}^{2}=2\left(\boldsymbol{U}, \frac{\mathrm{d} \boldsymbol{U}}{\mathrm{d} t}\right)_{h}=-\frac{2 A}{\Delta x}\left(\boldsymbol{V}, \mathrm{P}^{-1} \mathrm{Q} \boldsymbol{V}\right)_{h}
$$

Thanks to this relationship, if $\mathrm{P}^{-1} \mathrm{Q}$ is semibounded then it follows that

$$
\frac{\mathrm{d}}{\mathrm{d} t}\|\boldsymbol{U}\|_{h}^{2} \leq 2 \alpha\|\boldsymbol{U}\|_{h}^{2}
$$

which leads to

$$
\left\|\boldsymbol{U}^{n+1}\right\|_{h} \leq \mathrm{e}^{\alpha \Delta t}\left\|\boldsymbol{U}^{n}\right\|_{h}
$$

$\boldsymbol{U}^{n}$ and $\boldsymbol{U}^{n+1}$ being the solution vectors respectively at time $t^{n}$ and $t^{n+1}$. This results is stated in the following theorem.

Theorem 4.6. If the difference operator $\mathrm{P}^{-1} \mathrm{Q}$ is semibounded, then the problem (4.22) is energy stable, as in (4.27).

One can see that in this definition of energy stability a growth in time is allowed. To ensure stability for any $t \in] 0,+\infty)$, the scheme is required to be time energy stable.

Theorem 4.7. If the difference operator $\mathrm{P}^{-1} \mathrm{Q}$ is semibounded and satisfies

$$
-\frac{A}{\Delta x}\left(\boldsymbol{V}, \mathrm{P}^{-1} \mathrm{Q} \boldsymbol{V}\right)_{h} \leq 0,
$$

for any $\boldsymbol{V} \in \mathbb{R}^{n+1}$, then the approximation (4.22) is time energy stable.

It is clear that in this case the scheme satisfies

$$
\left\|\boldsymbol{U}^{n+1}\right\|_{h} \leq\left\|\boldsymbol{U}^{n}\right\|_{h} .
$$

A classical and commonly used class of schemes ensuring energy stability is the summation by parts (SBP) difference operators. Furthermore, these discretizations mimic the continuous level regarding the variation of energy. Indeed, defining the continuous energy as $\mathcal{E}(t)=\int_{0}^{1} u(x, t)^{2} \mathrm{~d} x$, it easily follows that

$$
\frac{\mathrm{d} \mathcal{E}}{\mathrm{d} t}=-A\left(u(1, t)^{2}-u(0, t)^{2}\right) .
$$

The SBP operators assume a norm matrix $\mathrm{H}$ such that

- the matrix HP is symmetric positive definite;

- the matrix $\mathrm{HQ}$ is nearly skew-symmetric such that $\mathrm{G}=\mathrm{HQ}+\mathrm{HQ}^{\mathrm{t}}$ admits only two elements: $G_{00}<0$ and $G_{n n}>0$. 
Doing so, the problem (4.22) admits a summation-by-part formula and the scheme is then energy stable. Defining the discrete energy as $E(t)=(\boldsymbol{U}, \boldsymbol{U})_{h}$, one easily gets

$$
\frac{\mathrm{d} E}{\mathrm{~d} t}=-\frac{2 A}{\Delta x}(\boldsymbol{U}, \mathrm{HQ} \boldsymbol{U})=-A\left(G_{n n} u_{n}^{2}+G_{00} u_{0}^{2}\right),
$$

which obviously proves the energy stability of the scheme, the difference operator being semibounded. One can also see in (4.30) that the discretization mimic the continuous energy behavior (4.29). However, the time stability issue remains. Considering the case where $x_{0}$ is an inflow boundary and $x_{n}$ is an outflow boundary $(A>0)$, and introducing the prescribed boundary condition $u(0, t)=g(t)$, which can be set to zero for the purpose of stability, the continuous energy rewrites

$$
\frac{\mathrm{d} \mathcal{E}}{\mathrm{d} t}=-A u(1, t)^{2} \leq 0 .
$$

We see that in (4.30), nothing ensures the time rate of change of the discrete energy to be less than zero. A solution to overcome this problem is the use of a simultaneous approximation term (SAT), see [1]. In this case, a penalty term is used to take into account the inflow boundary contribution and ensure time stability. For instance, a SBP-SAT discretization of the considered problem would write

$$
\mathrm{P} \frac{\mathrm{d} \boldsymbol{U}}{\mathrm{d} t}=-\frac{A}{\Delta x}\left(\mathrm{Q} \boldsymbol{U}-\tau G_{00}\left(u_{0}-g(t)\right) \boldsymbol{S}\right),
$$

where $\boldsymbol{S}=\mathrm{H}^{-1}(1,0, \ldots, 0)^{\mathrm{t}}$. For the time stability analysis, we take as before $g(t)=0$. Using the difference operator (4.32), the time rate of change of the discrete energy now writes

$$
\frac{\mathrm{d} E}{\mathrm{~d} t}=-A\left(G_{n n} u_{n}^{2}-2 G_{00}\left(\tau-\frac{1}{2}\right) u_{0}^{2}\right) .
$$

We can immediately state that the SBP-SAT discretization (4.32) is both stable and time-stable if $\tau \geq \frac{1}{2}$.

We have seen here that to ensure time energy stability, taking into account the inflow boundary condition, specific modifications of the difference scheme is required, see $[1,7,19]$. Actually, all these constraints vanish using the inverse Lax-Wendroff inflow boundary treatment. This noteworthy advantage results from the fact that using ILW, all the ghost points can be identified as prescribed boundary conditions. As we have seen in Section 4.1, in the case where $g(t)=0$, the ghost points contribution is naturally null. Thus, the inner scheme remains unchanged near the inflow boundary. The SBP discretization using ILW for the inflow boundary will perfectly corresponds to the SBP operator obtained in the case of the outflow quarter plan problem with a finite number of grid points. In this case, a norm matrix $\mathrm{H}$ ensuring the following assumptions is considered

- the matrix HP is symmetric positive definite,

- the matrix $\mathrm{HQ}$ is nearly skew-symmetric such that $\mathrm{G}=\mathrm{HQ}+\mathrm{HQ}^{\mathrm{t}}$ admits only one element, $G_{n n}>0$.

As mentioned before, the scheme remains unchanged near the inflow boundary. Using such a scheme yields the following time rate of change of the discrete energy

$$
\frac{\mathrm{d} E}{\mathrm{~d} t}=-A G_{n n} u_{n}^{2} \leq 0,
$$

which ensure time stability of the scheme, and is furthermore consistent with the continuous level result (4.31). This relationship holds even if the first grid point is not aligned with the inflow boundary. This result exhibits the high ability of the ILW boundary treatment to mimic naturally the behavior of the continuous solution, considering ghost points as physical boundaries. If we consider the simplified inverse Lax-Wendroff boundary treatment, things will be different. One may still be able to find the appropriate norm ensuring energy stability when the simplified inverse Lax-Wendroff boundary treatment is used. However it may be expected that the 
moments to be extrapolated would need to be extrapolated in a specific way, using a wider stencil, than what has been used in the previous sections. Since the energy method provides only a sufficient condition for stability, it may not be able to determine the minimum number of leading terms required in the procedure to ensure stability. For this reason we have tackled stability in this paper for the simplified inverse Lax-Wendroff boundary treatment using the G-K-S theory only.

\section{Conclusion}

In this paper, we have assessed the stability of the central compact schemes introduced recently in [14], provided with particular boundary conditions. For the outflow boundary, the case of classical Lagrangian extrapolation has been studied. Regarding the inflow boundary, the case of the inverse Lax-Wendroff boundary treatment, see [23], and a simplified version of it as used in [24] have been addressed. Finally, the outflow extrapolation and inflow inverse Lax-Wendroff boundary treatments have both proved to maintain stability and the order of accuracy, under the same CFL condition as that for the periodic boundary case, for any central compact schemes presented and for any position of the boundary. For the simplified inverse Lax-Wendroff boundary treatment, stability depends on the number of leading terms $k_{d}$ used in the procedure. Nevertheless, SILW maintains stability of central compact schemes under the same CFL as that the periodic boundary in the case when enough terms are used, otherwise the scheme is not stable for any CFL condition. The minimum number of leading terms required in the SILW inflow boundary condition by different central compact schemes to remain stable, using the third-order TVD Runge-Kutta as time discretization, has been summarized in Table 1. Finally, the natural suitability of the inverse Lax-Wendroff inflow boundary to the energy stability considerations has been enlightened.

An important result which came out is the perfect consistency in the results obtained by the two different analysis, G-K-S and eigenspectrum visualization. On the one hand, in the G-K-S theory the stability analysis is broken into three simpler problems, the inner problem and the two quarter-plane problems corresponding to the two type of boundaries, inflow and outflow. On the other hand, the matrix operator we compute the eigenvalues of, in the spectrum analysis, contains both boundary conditions. But due to the fact that it has been proven that no instability comes from the outflow extrapolation procedure, if one observes in the spectra some unstable eigenvalues it is clear that they come from the inflow contribution, and thus correspond to the ones found with G-K-S theory and normal mode analysis.

In the future, we plan to investigate furthermore the stability analysis of the considered schemes using the energy method in the case of SILW inflow boundary. As said previously, such method has a simple structure and is convenient to use. The drawback is that if one fails to construct such a norm, it is not clear whether or not the scheme is stable. In the case of very high order compact schemes, building such a norm is not an easy task. Another interesting issue could be to adapt central compact schemes and inverse Lax-Wendroff boundary treatment to SBP-SAT operators. The summation by parts (SBP) operators provide inherently a norm ensuring stability. By adding a simultaneous approximation term (SAT), the boundary condition is naturally taken into account. Applications of the studied schemes and boundary treatments to more practical problems, as for example the Euler or Navier-Stokes system of equations, are also planned.

\section{REFERENCES}

[1] M. Carpenter, D. Gottlieb and S. Abarbanel, Time-stable conditions for finite-difference schemes solving hyperbolic systems: methodology and application to high-order compact schemes. J. Comput. Phys. 111 (1994) 220-236.

[2] M. Carpenter, J. Nordström and D. Gottlieb, A stable and conservative interface treatment of arbitrary spatial accuracy. $J$. Comput. Phys. 148 (1999) 341-365.

[3] G.S. Constantinescu and S.K. Lele, Large eddy simulation of a near sonic turbulent jet and its radiated noise. AIAA Paper 0376 (2001).

[4] M. Goldberg, On a boundary extrapolation theorem by Kreiss. Math. Comput. 31 (1977) 469-477.

[5] M. Goldberg and E. Tadmor, Scheme-independent stability criteria for difference approximations of hyperbolic initial-boundary value problems. I. Math. Comput. 32 (1978) 1097-1107. 
[6] M. Goldberg and E. Tadmor, Scheme-independent stability criteria for difference approximations of hyperbolic initial-boundary value problems. II. Math. Comput. 36 (1981) 603-626.

[7] B. Gustafsson, On the implementation of boundary conditions for the methode of lines. BIT 38 (1998) $293-314$.

[8] B. Gustafsson, H.-O. Kreiss and J. Oliger, Time dependent problems and difference methods. John Wiley \& Sons (1995).

[9] B. Gustafsson, H.-O. Kreiss and A. Sundström, Stability theory of difference approximations for mixed initial boundary value problem. II. Math. Comput. 26 (1972) 649-686.

[10] H.-O. Kreiss and G. Scherer, Finite element and finite difference methods for hyperbolic partial differential operators. Mathematical aspects of finite elements in partial differential equations. Academic Press, Orlando, FL (1974).

[11] H.-O. Kreiss and G. Scherer, On the existence of energy estimates for difference approximations for hyperbolic systems. Technical report, Uppsala University, Sweden (1977).

[12] S. Lee, S.K. Lele and P. Moin, Interaction of isotropic turbulence with shock waves: effect of shock strength. J. Fluid Mech. 340 (1997) 225-247.

[13] S.K. Lele, Compact finite difference schemes with spectral-like resolution. J. Comput. Phys. 103 (1992) $16-42$.

[14] X. Liu, S. Zhang, H. Zhang and C.-W. Shu, A new class of central compact schemes with spectral-like resolution I: linear schemes. J. Comput. Phys. 248 (2013) 235-256.

[15] K. Mahesh, S.K. Lele and P. Moin, The influence of entropy fluctuation on the interaction of turbulence with a shock wave. J. Fluid Mech. 334 (1997) 353-379.

[16] K. Mattsson, Boundary procedure for summation-by-parts operators. J. Sci. Comput. 18 (2003) 133-153.

[17] K. Mattsson and J. Nordström, Summation by parts operators for finite difference approximations of second derivatives. J. Comput. Phys. 199 (2004) 503-540.

[18] P. Moin, K. Squires, W. Cabot and S. Lee, A dynamic subgridscale model for compressible turbulence and scalar transport. Phys. Fluid 3 (1991) 2746-2757.

[19] P. Olson, Summation by parts, projection and stability: I. Math. Comput. 64 (1995) 1035-1065.

[20] C.-W. Shu and S. Osher, Efficient implementation of essentially non-oscillatory shock-capturing schemes. J. Comput. Phys. 77 (1988) 439-471.

[21] B. Strand, Summation by parts for finite difference approximations for d/dx. J. Comput. Phys. 110 (1994) $47-67$.

[22] J.C. Strikwerda, Initial boundary value problems for the method of lines. J. Comput. Phys. 34 (1980) 94-107.

[23] S. Tan and C.-W. Shu, Inverse Lax-Wendroff procedure for numerical boundary conditions of conservations laws. J. Comput. Phys. 229 (2010) 8144-8166.

[24] S. Tan, C. Wang, C.-W. Shu and J. Ning, Efficient implementation of high order inverse Lax-Wendroff boundary treatment for conservation laws. J. Comput. Phys. 231 (2012) 2510-2527. 